\title{
Primary Immunodeficiency Diseases: an Update on the Classification from the International Union of Immunological Societies Expert Committee for Primary Immunodeficiency 2015
}

\author{
Capucine Picard $^{1,2}$ - Waleed Al-Herz ${ }^{3,4}$ • Aziz Bousfiha ${ }^{5}$ - Jean-Laurent Casanova ${ }^{1,6,7,8,9}$. \\ Talal Chatila ${ }^{10}$ • Mary Ellen Conley ${ }^{6}$. Charlotte Cunningham-Rundles ${ }^{11}$. \\ Amos Etzioni ${ }^{12}$ • Steven M. Holland ${ }^{13}$. Christoph Klein ${ }^{14}$ • Shigeaki Nonoyama ${ }^{15}$. \\ Hans D. Ochs ${ }^{16}$ • Eric Oksenhendler ${ }^{17,18}$ • Jennifer M. Puck ${ }^{19}$ - Kathleen E. Sullivan ${ }^{20}$ • \\ Mimi L K. Tang ${ }^{21,22,23}$ • Jose Luis Franco ${ }^{24}$ H. Bobby Gaspar ${ }^{25}$
}

Received: 20 July 2015 / Accepted: 20 September 2015 /Published online: 19 October 2015

(C) The Author(s) 2015. This article is published with open access at Springerlink.com

\begin{abstract}
We report the updated classification of primary immunodeficiencies compiled by the Primary Immunodeficiency Expert Committee (PID EC) of the International Union of Immunological Societies (IUIS). In the two years since the
\end{abstract}

H. Bobby Gaspar

h.gaspar@ucl.ac.uk

1 Laboratory of Human Genetics of Infectious Diseases, Necker Branch, INSERM UMR1163, Necker Hospital for Sick Children, Paris, France

2 Centre d'étude des déficits immunitaires (CEDI), Hôpital Necker-Enfants Malades, AP-HP, Paris, France

3 Department of Pediatrics, Faculty of Medicine, Kuwait University, Kuwait City, Kuwait

4 Allergy and Clinical Immunology Unit, Department of Pediatrics, Al-Sabah Hospital, Kuwait City, Kuwait

5 Clinical Immunology Unit, Casablanca Children's Hospital, Ibn Rochd Medical School, King Hassan II University, Casablanca, Morocco

6 St. Giles Laboratory of Human Genetics of Infectious Diseases, Rockefeller Branch, The Rockefeller University, New York, NY, USA

7 Howard Hughes Medical Institute, New York, NY, USA

8 University Paris Descartes, Imagine Institute, Paris, France

9 Pediatric Hematology \& Immunology Unit, Necker Hospital for Sick Children, Paris, France

10 Division of Immunology, Children's Hospital Boston, Boston, MA, USA

11 Department of Medicine and Pediatrics, Mount Sinai School of Medicine, New York, NY, USA previous version, 34 new gene defects are reported in this updated version. For each disorder, the key clinical and laboratory features are provided. In this new version we continue to see the increasing overlap between immunodeficiency, as

12 Meyer Children's Hospital-Technion, Haifa, Israel

13 Laboratory of Clinical Infectious Diseases, National Institute of Allergy and Infectious Diseases, Bethesda, MD, USA

14 Dr von Hauner Children's Hospital, Ludwig-Maximilians-University Munich, Munich, Germany

15 Department of Pediatrics, National Defense Medical College, Saitama, Japan

16 Department of Pediatrics, University of Washington and Seattle Children's Research Institute, Seattle, WA, USA

17 Department of Clinical Immunology, Hôpital Saint-Louis, Assistance Publique-Hôpitaux de Paris, Paris, France

18 Université Paris Diderot, Sorbonne Paris Cité, Paris, France

19 Department of Pediatrics, University of California San Francisco and UCSF Benioff Children's Hospital, San Francisco, CA, USA

20 Division of Allergy Immunology, Department of Pediatrics, The Children's Hospital of Philadelphia, Philadelphia, PA, USA

21 Murdoch Childrens Research Institute, Melbourne, VIC, Australia

22 Department of Paediatrics, University of Melbourne, Melbourne, VIC, Australia

23 Department of Allergy and Immunology, Royal Children's Hospital, Melbourne, Australia

24 Group of Primary Immunodeficiencies, University of Antioquia, Medellin, Colombia

25 UCL Institute of Child Health, 30, Guilford Street, London WC1N 1EH, UK 
manifested by infection and/or malignancy, and immune dysregulation, as manifested by auto-inflammation, auto-immunity, and/or allergy. There is also an increased number of genetic defects that lead to susceptibility to specific organisms which reflects the finely tuned nature of immune defense systems. This classification is the most up to date catalogue of all known and published primary immunodeficiencies and acts as a current reference of the knowledge of these conditions and is an important aid for the genetic and molecular diagnosis of patients with these rare diseases.

Keywords Primary immunodeficiencies · classification · genetic defects

\section{Background}

The International Union of Immunological Societies (IUIS) Expert Committee on Primary Immunodeficiency met in London on the 14th and 15th March 2015 to update the classification of human primary immunodeficiencies (PIDs). This report represents the most current and complete catalogue of known PIDs. It serves as a reference for these conditions and provides a framework to help in the diagnostic approach to patients suspected to have PID.

As in previous reports, we have classified the conditions into major groups of PIDs and these are now represented in 9 different tables (Tables 1, 2, 3, 4, 5, 6, 7,8 and 9 ). In each table, we list the condition, its genetic defect if known and the major immunological and in some conditions the non-immunological abnormalities associated with the disease. This year we have added the gene OMIM number as well as the phenotype OMIM number for ease of reference.

The classification this year differs in a number of ways from the previous edition published in 2014. Importantly, each defect is now listed in only one table. The diverse immunological phenotypes of many conditions imply that a very large number of conditions could very readily be listed in multiple tables. However, with the increasing number of identified defects, this would make each table large and cumbersome. For this reason, we chose to list each defect in one table only and to place it according to the most pronounced and fundamental defect. For this reason and as an example, CD40L deficiency is now found in Table 1 amongst combined immunodeficiencies, because $\mathrm{CD} 40 \mathrm{~L}$ is a $\mathrm{T}$ cell signaling molecule whose absence leads to both cellular and humoral defects, even though it was originally described as an antibody deficiency. Although some of our placements may be disputed, the committee came to these decisions after much thought and deliberation.
The title of Table 6 has now been slightly changed to 'Defects in intrinsic and innate immunity' and contains defects characterized by susceptibility to specific organisms. For this reason, the MSMDs (Mendelian Susceptibility to Mycobacterial Disease) are now in Table 6, having previously been in Table 5 (Phagocytic Disorders).

In previous editions, we have placed an asterisk against conditions in which 10 or fewer individuals had been described in the literature. However, this is now felt to be an artificial indicator as, once described, a condition may be found in additional patients but not necessarily reported. For this reason, there is no specific indicator of the number of patients identified or reported.

There is a growing appreciation of wide phenotypic variability for many of the individual specific gene defects, reflecting not only the variety of mutations within each gene but also host and/or environmental modifying factors that may impact the phenotype even between individuals with the same mutation within the same gene. The complexities of these conditions in terms of clinical and immunological presentation and heterogeneity cannot easily be captured in the limited space of a table format. For this reason, the furthest right column contains the Online Mendelian Inheritance in Man (OMIM) reference for each condition to allow access to a source of greater detail and updated information as to the phenotype.

A number of the new genes included in this edition of the classification tables are molecules associated not only with the immune system, but also with more generic cellular functions; such defects result in both immunological and nonimmunological abnormalities. In addition, there are a number of gain-of-function (GOF) mutations identified such as in PIK3CD. In CARD11 and STAT1 for example, there are both autosomal dominant GOF and autosomal recessive loss of function variants and these different modes of inheritance in the same gene lead to different functional consequences and hence different immunological and clinical phenotypes. The other trend that is increasingly observed is the increase in disorder of immunedysregulation rather than pure immunodeficiency.

The goal of the IUIS Expert Committee on Primary Immunodeficiencies is to increase awareness, facilitate recognition and promote optimal treatment for patients with Primary Immunodeficiencies. In addition to the current report and previous 'classification table' publications, the committee has also produced a 'Phenotypic Approach for IUIS PID classification and Diagnosis: Guidelines for Clinicians at the Bedside,' which aims to lead physicians to particular groups of PIDs starting from clinical features and combining routine immunological investigations. This will be further updated to include the newly identified defects. Together these contributions will hopefully allow a practical clinical framework for PID diagnosis. 


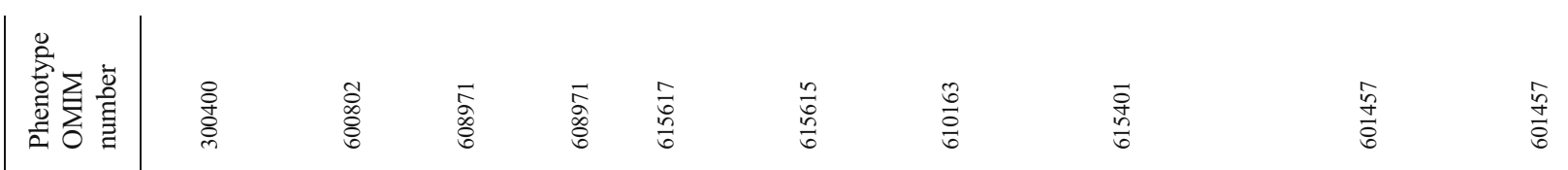

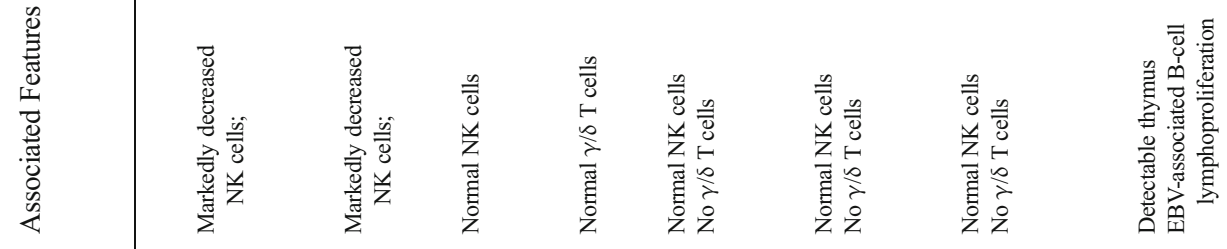

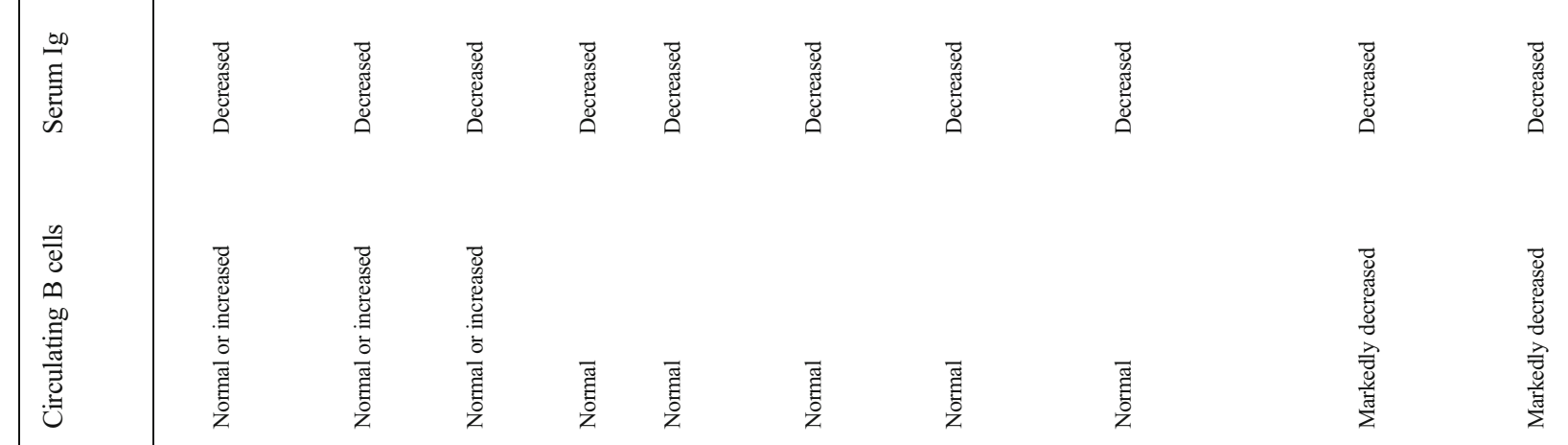

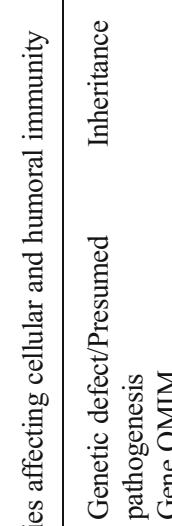

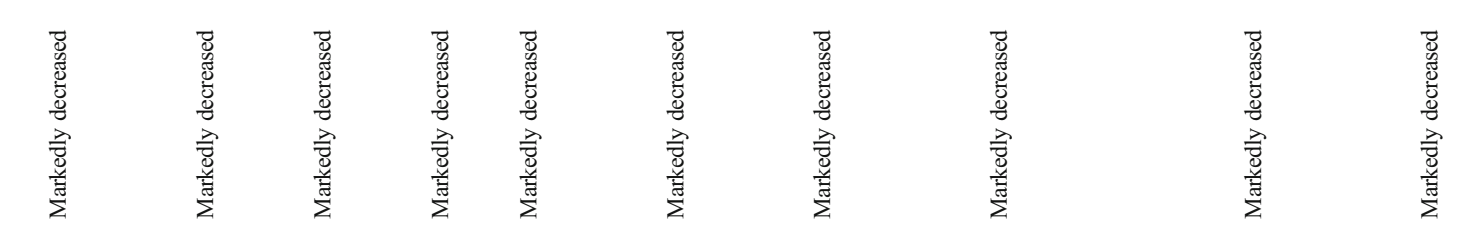

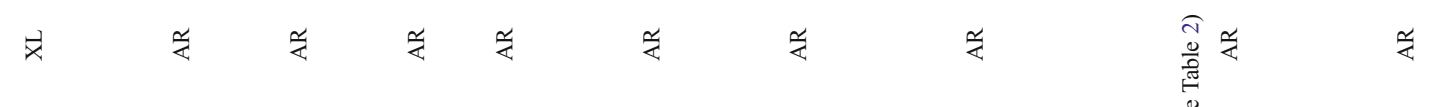

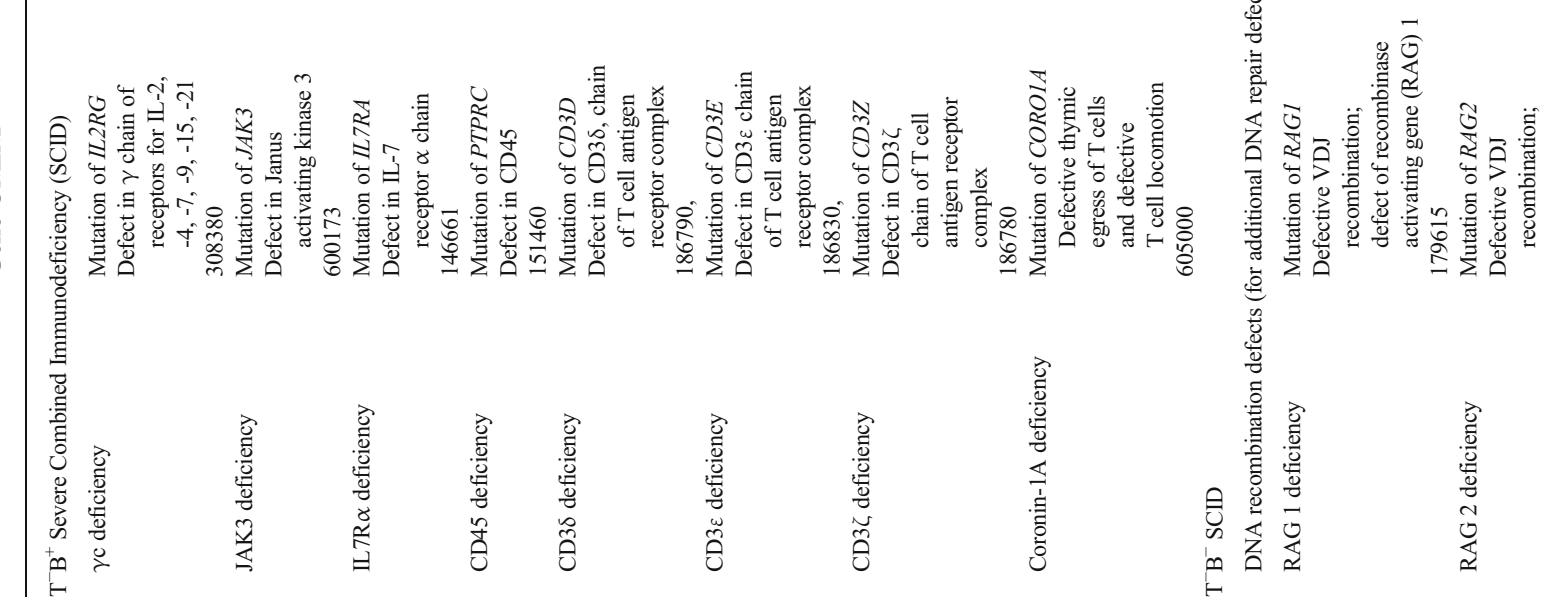




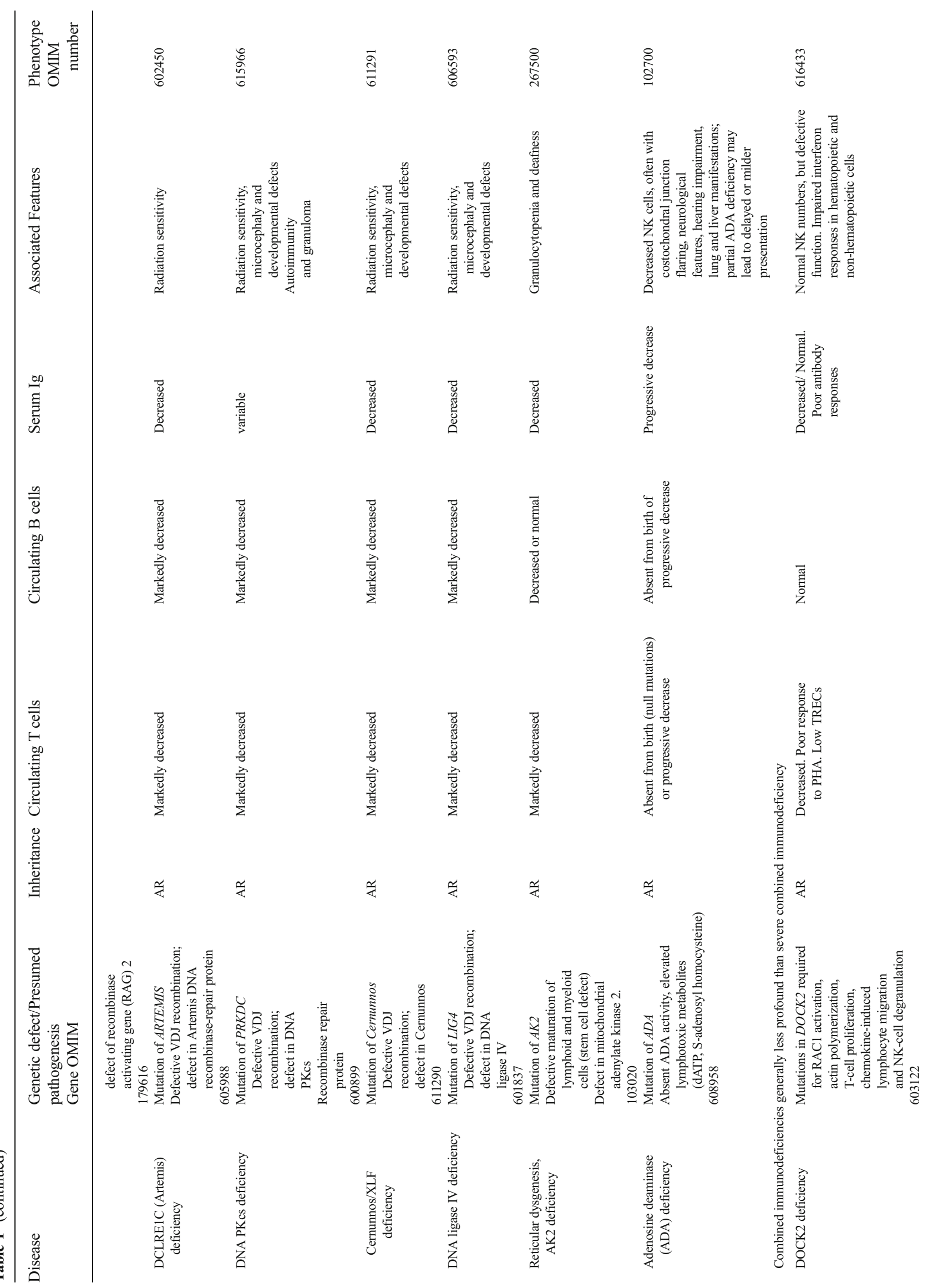




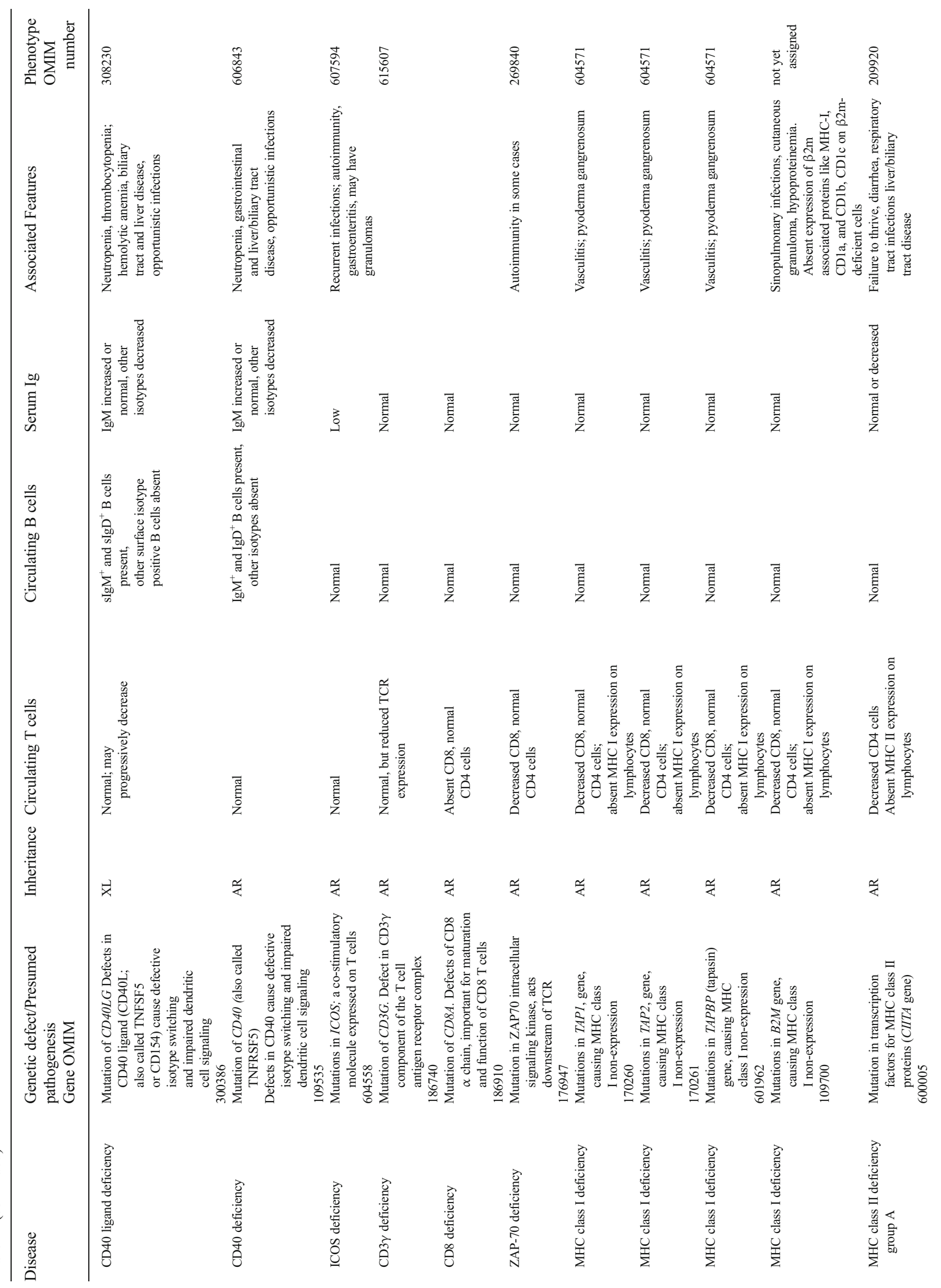




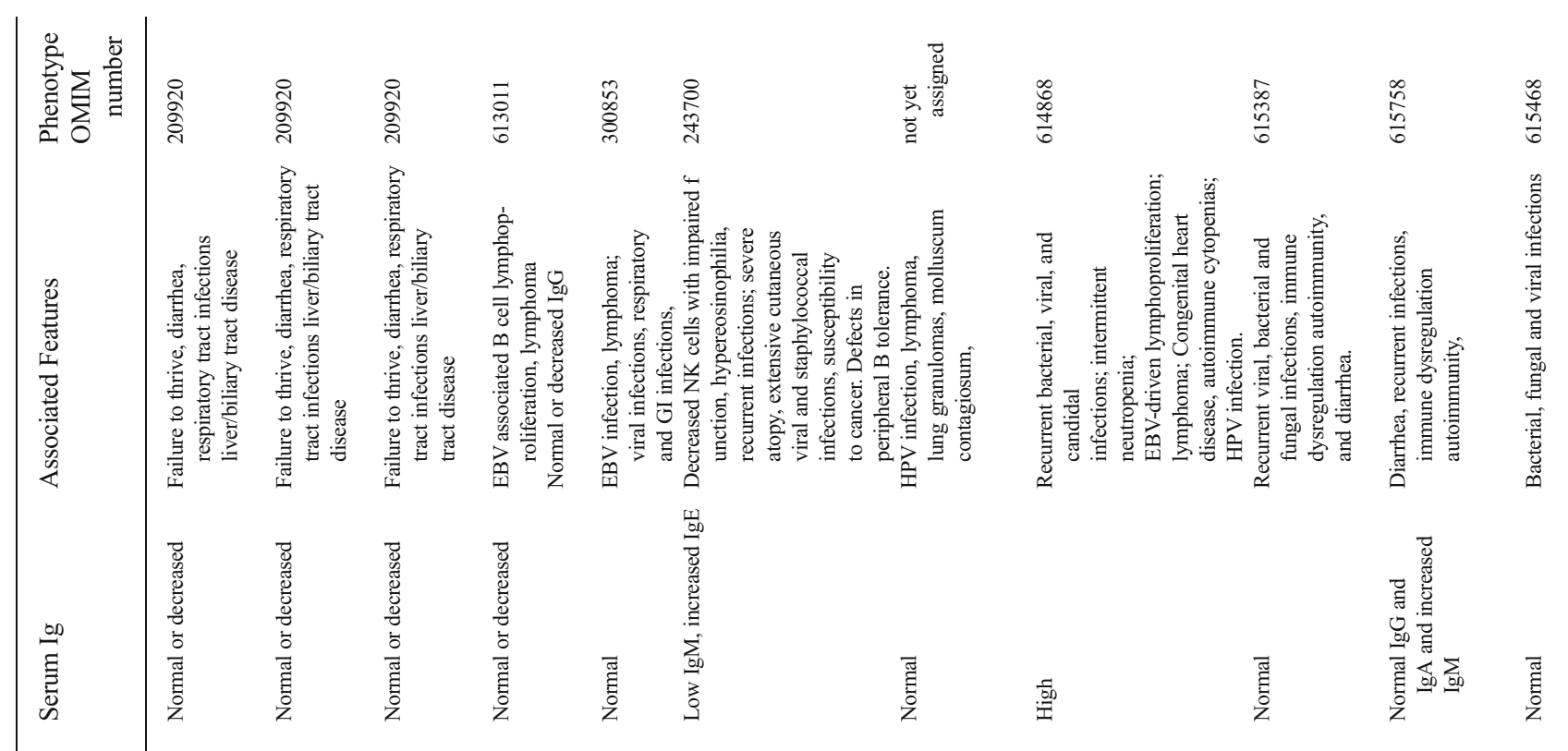

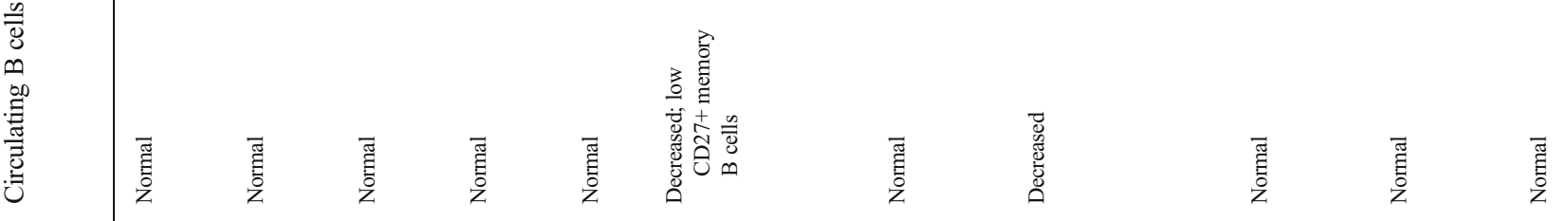

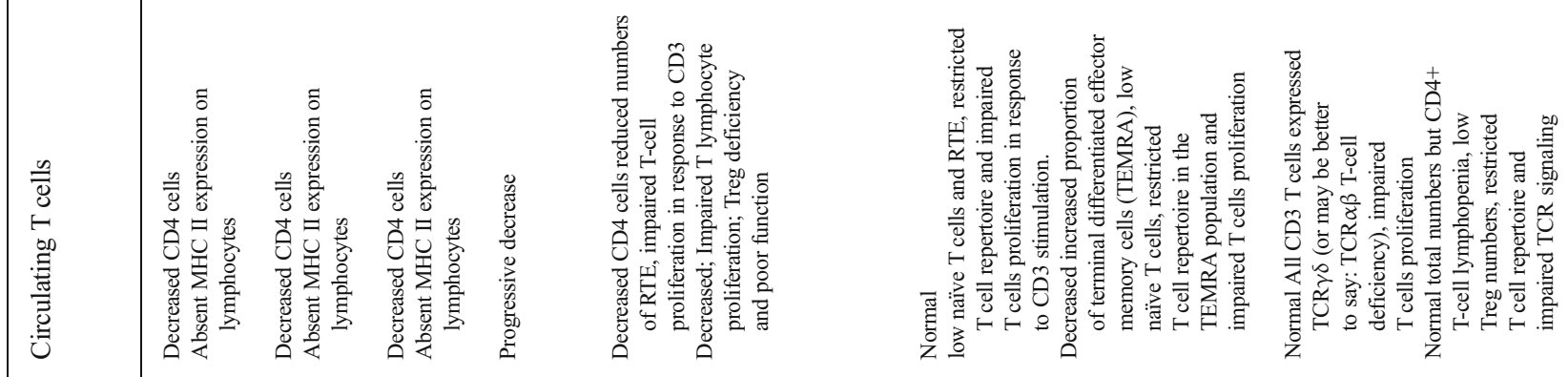

苞

$\cong \quad \cong \quad \because \quad \approx \quad \approx$

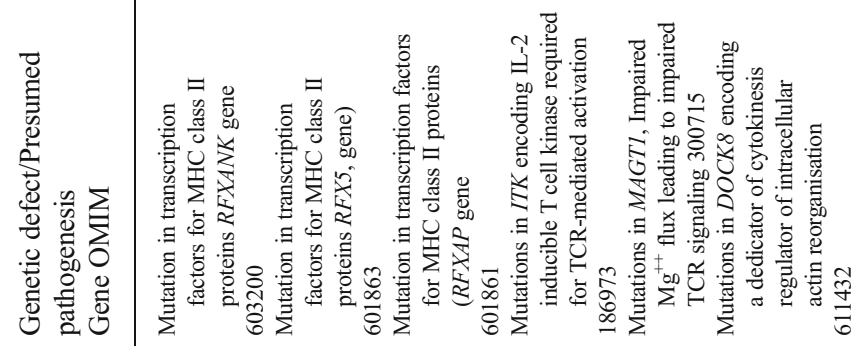

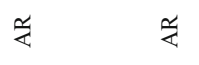

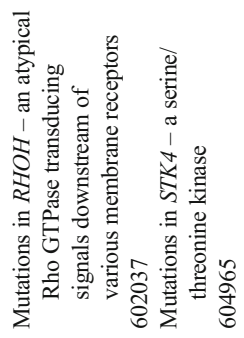

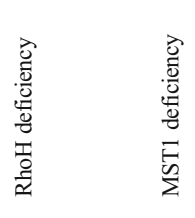

$\cong \quad \cong$

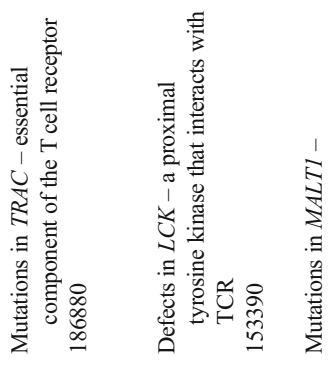

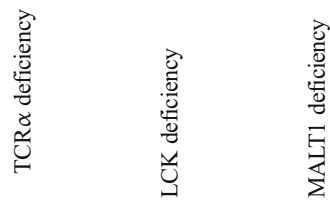




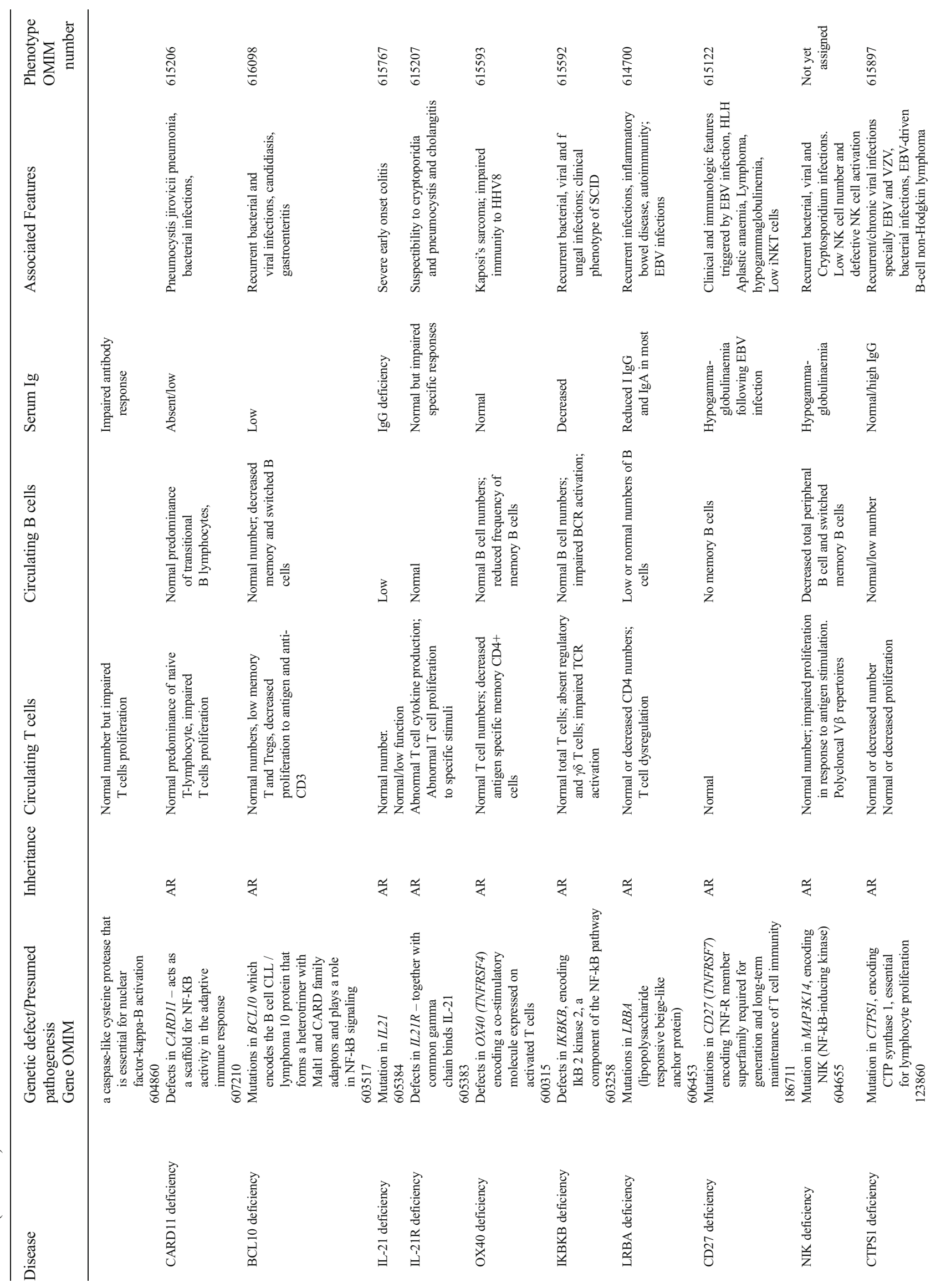




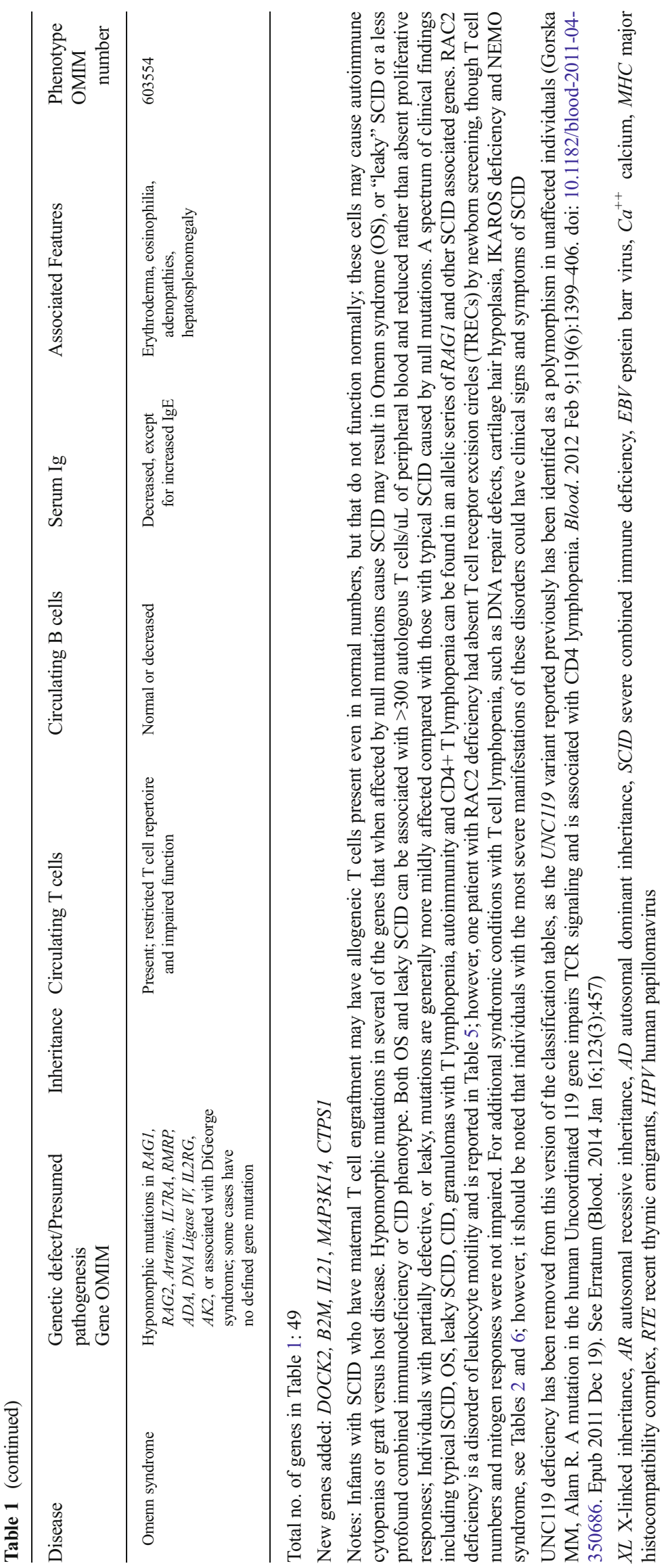




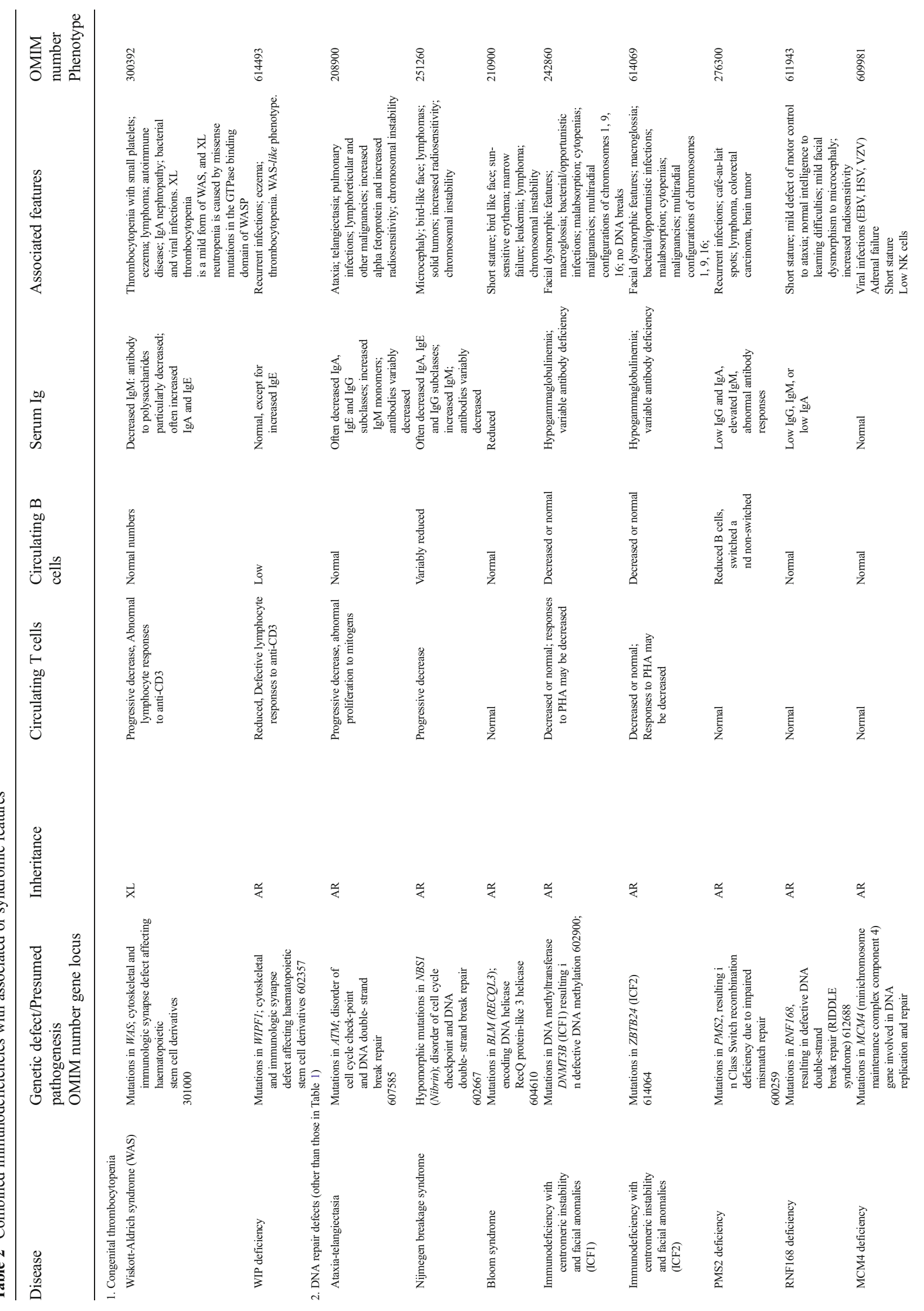




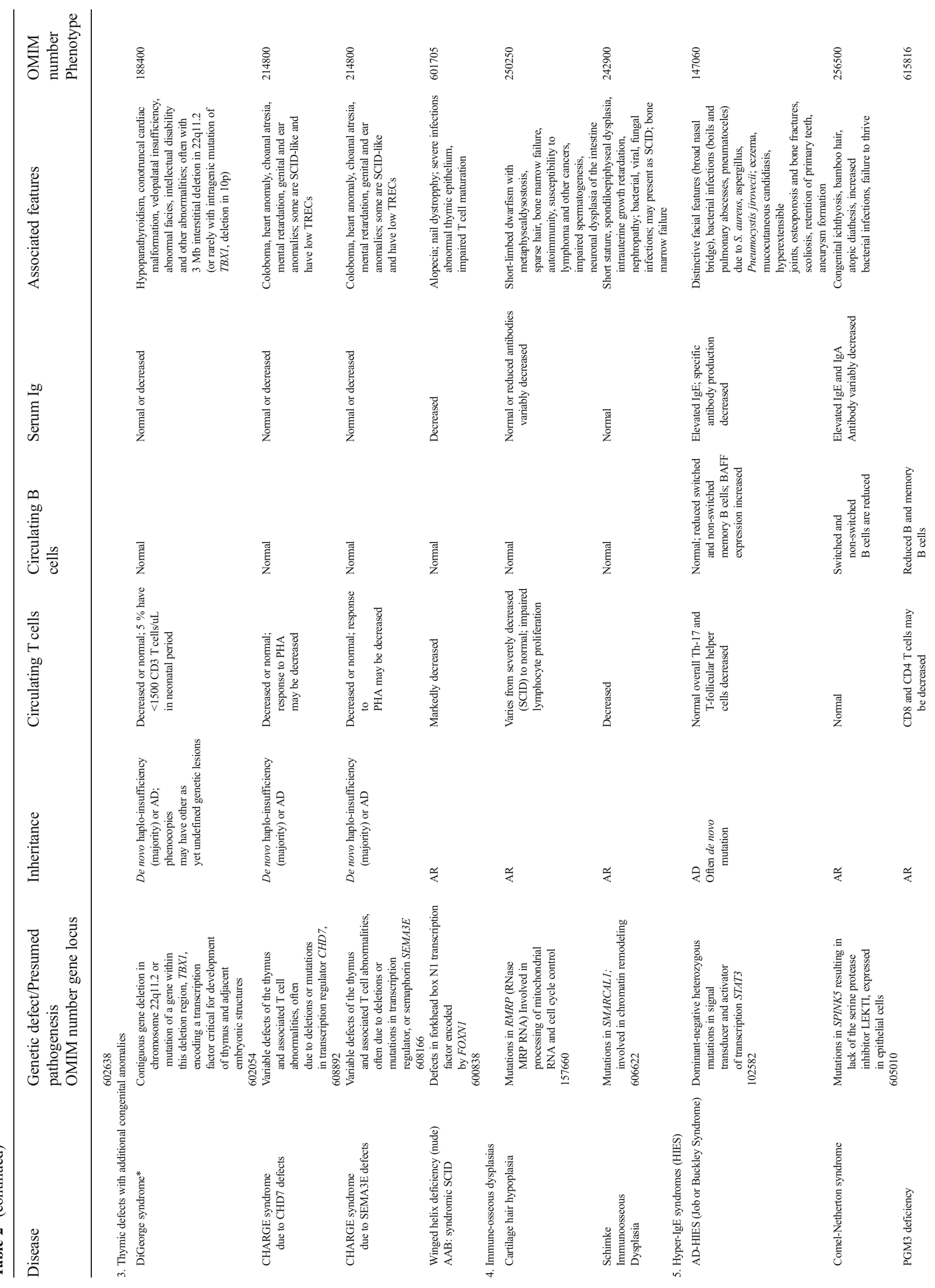




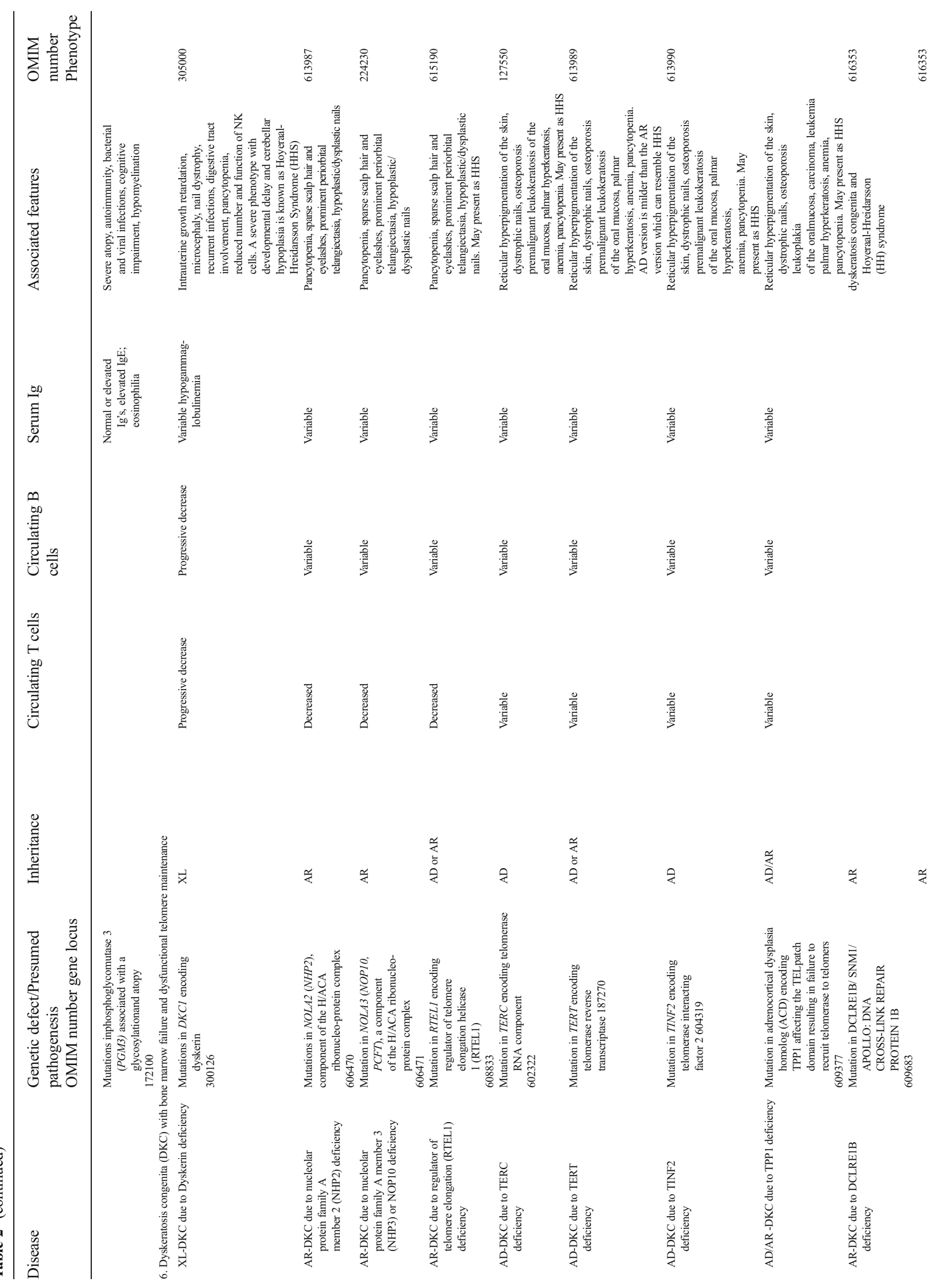




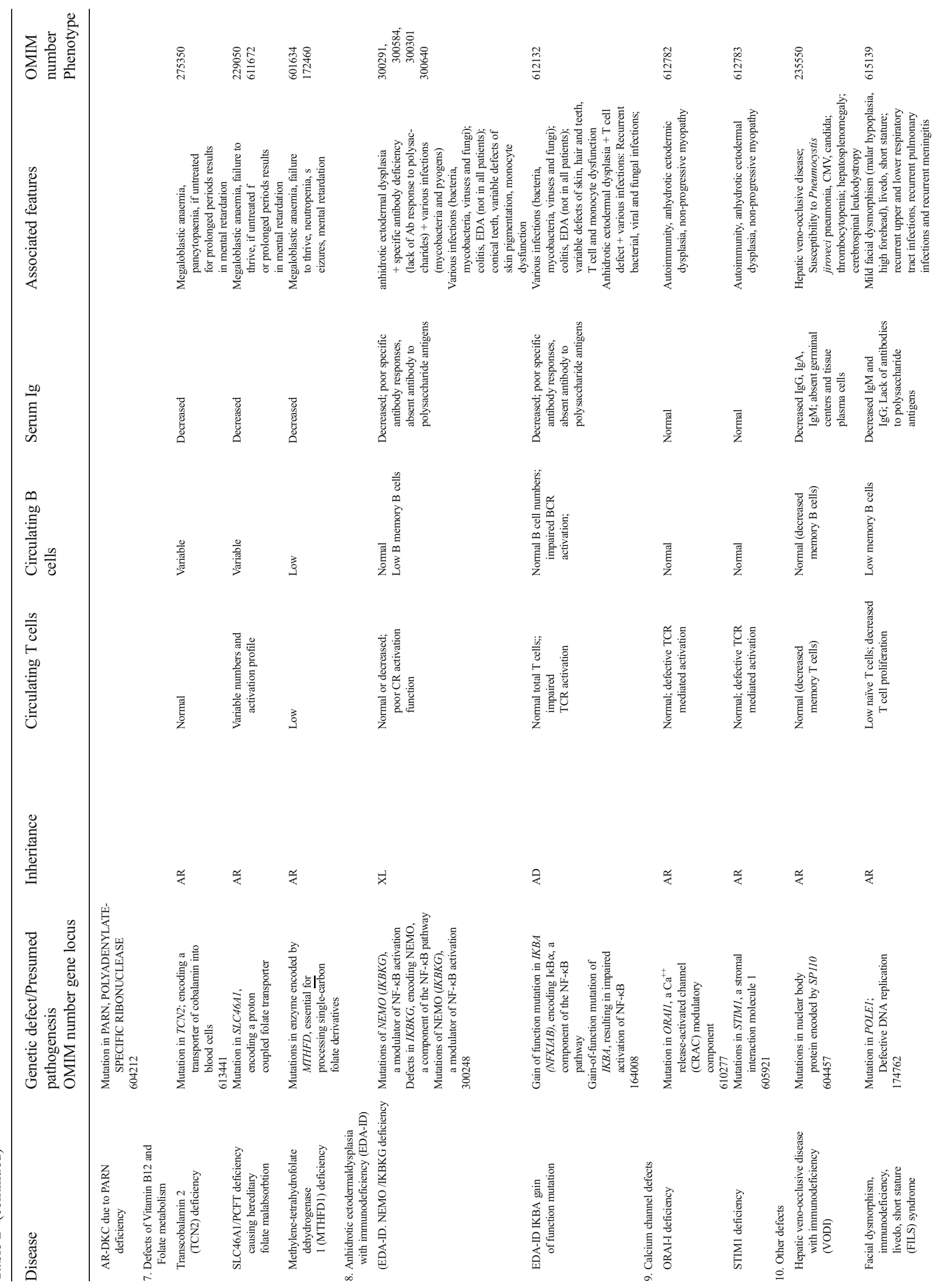




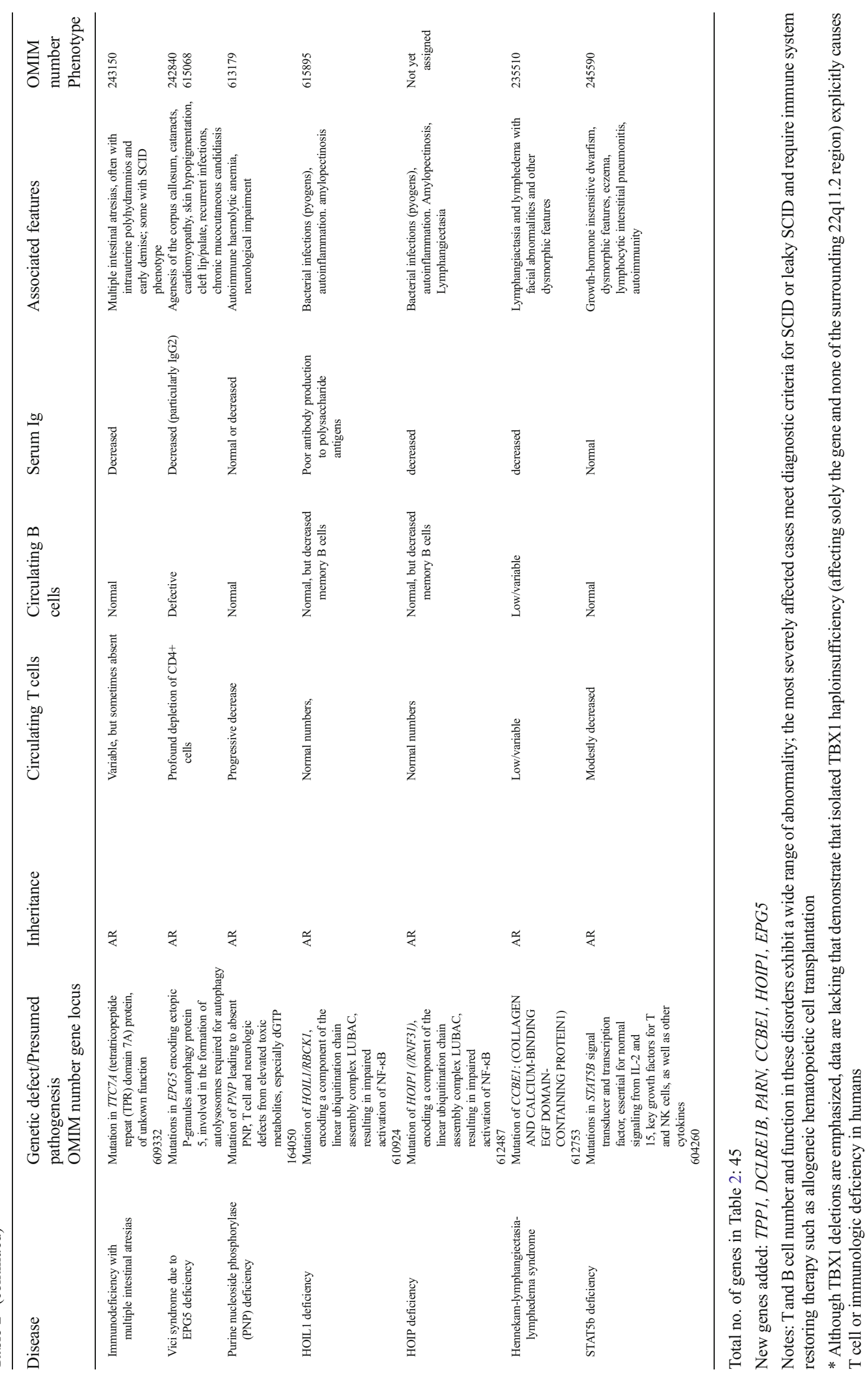




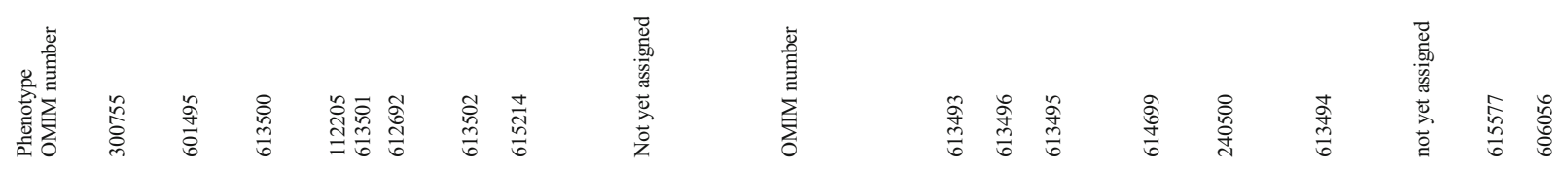

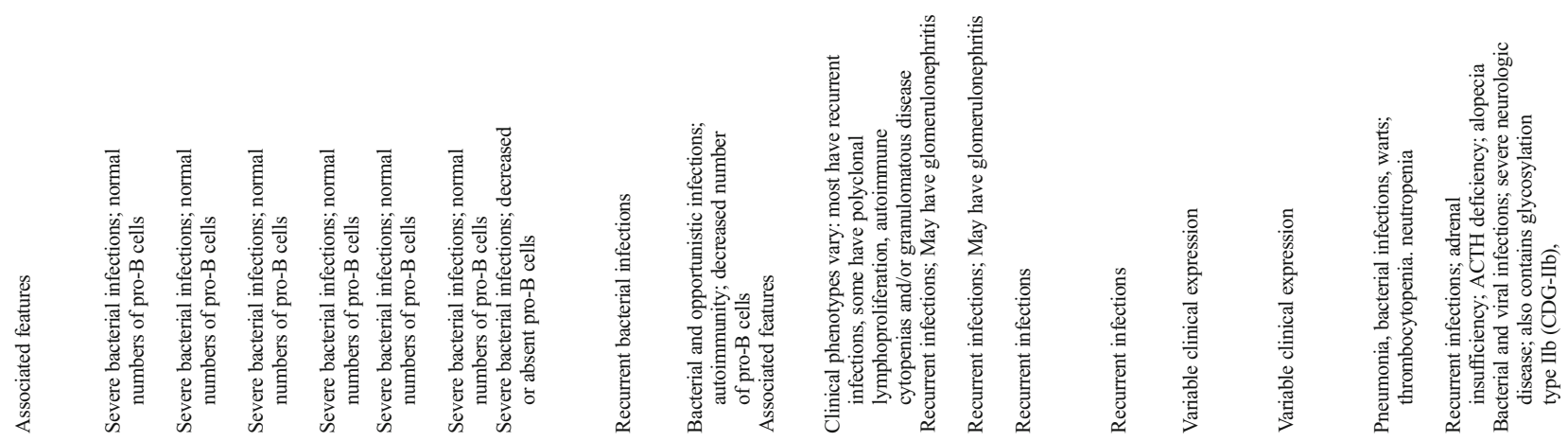

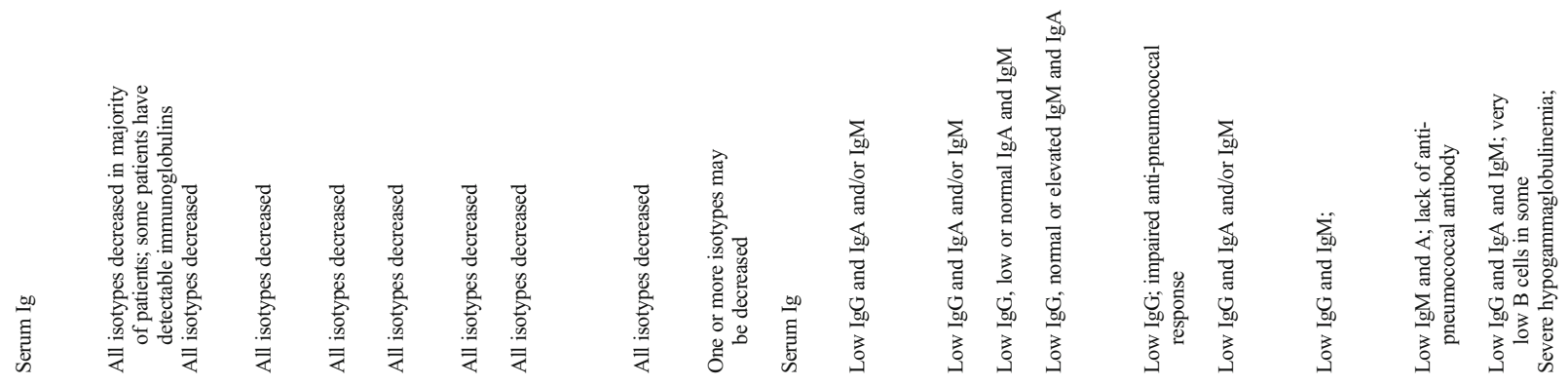

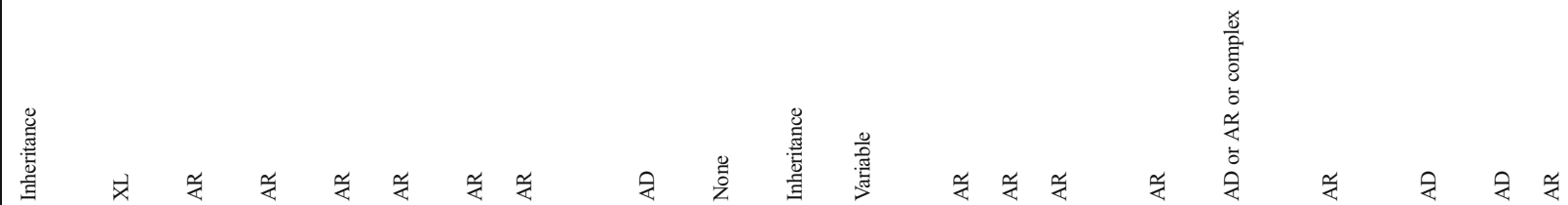
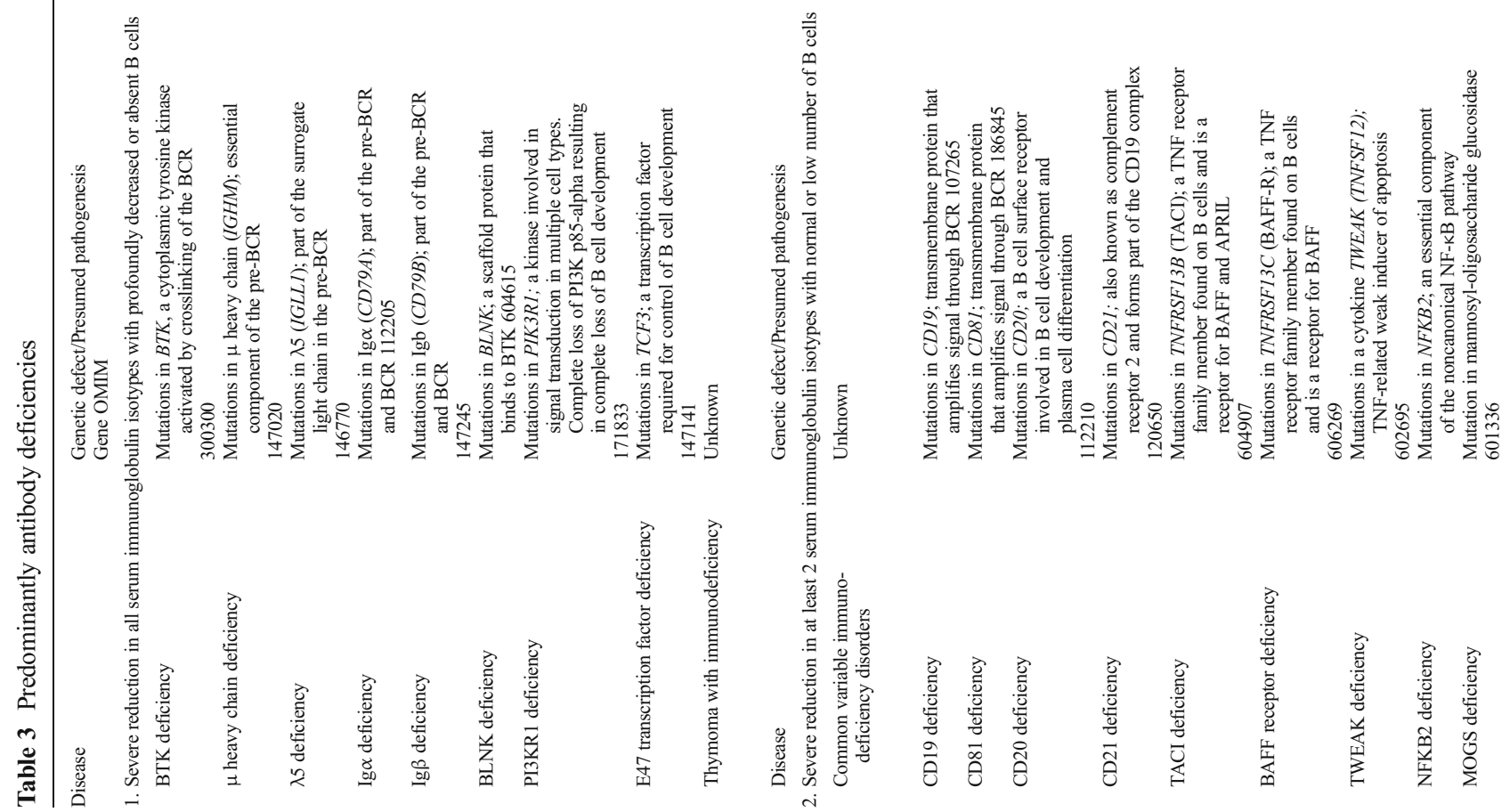


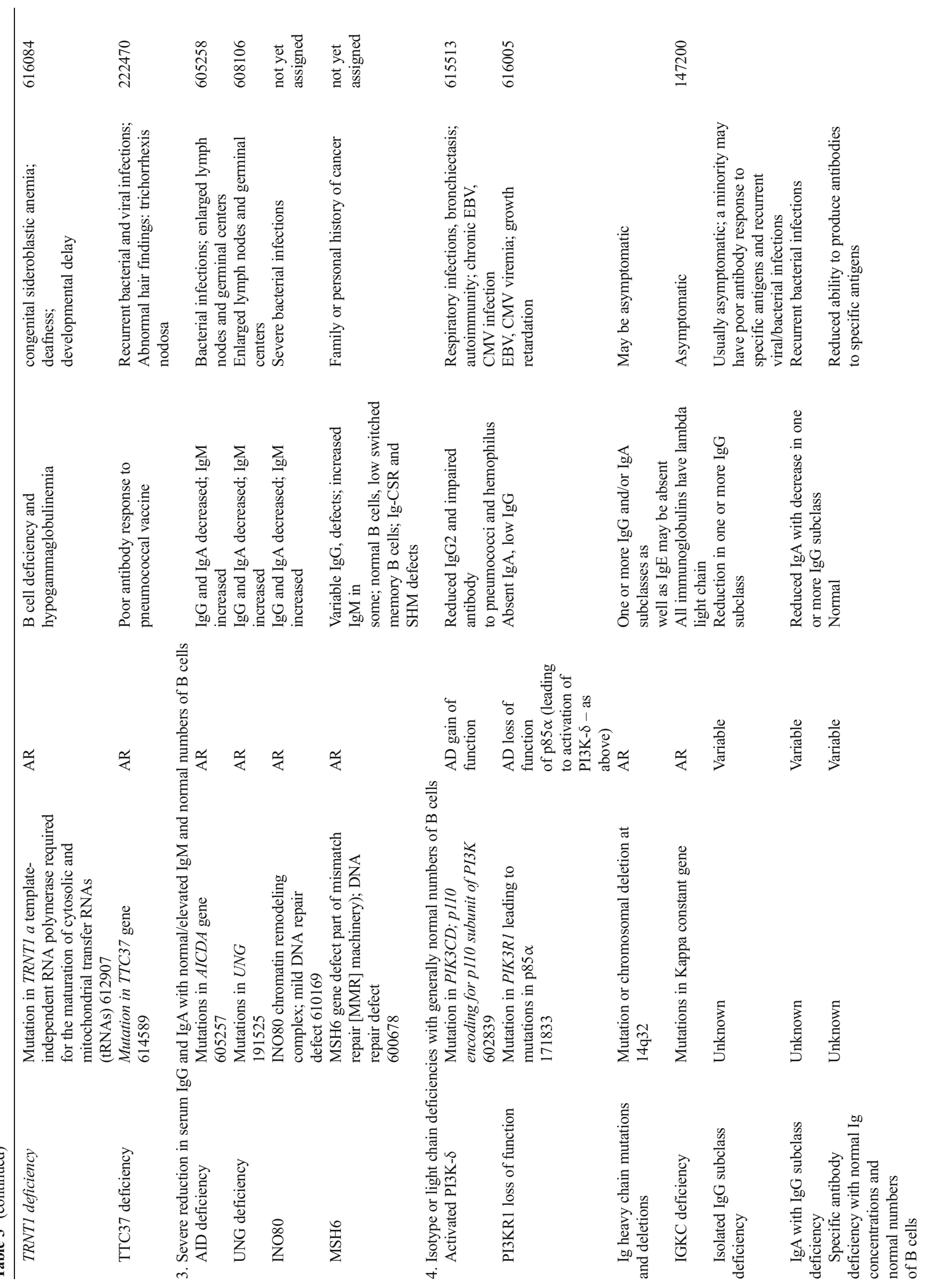




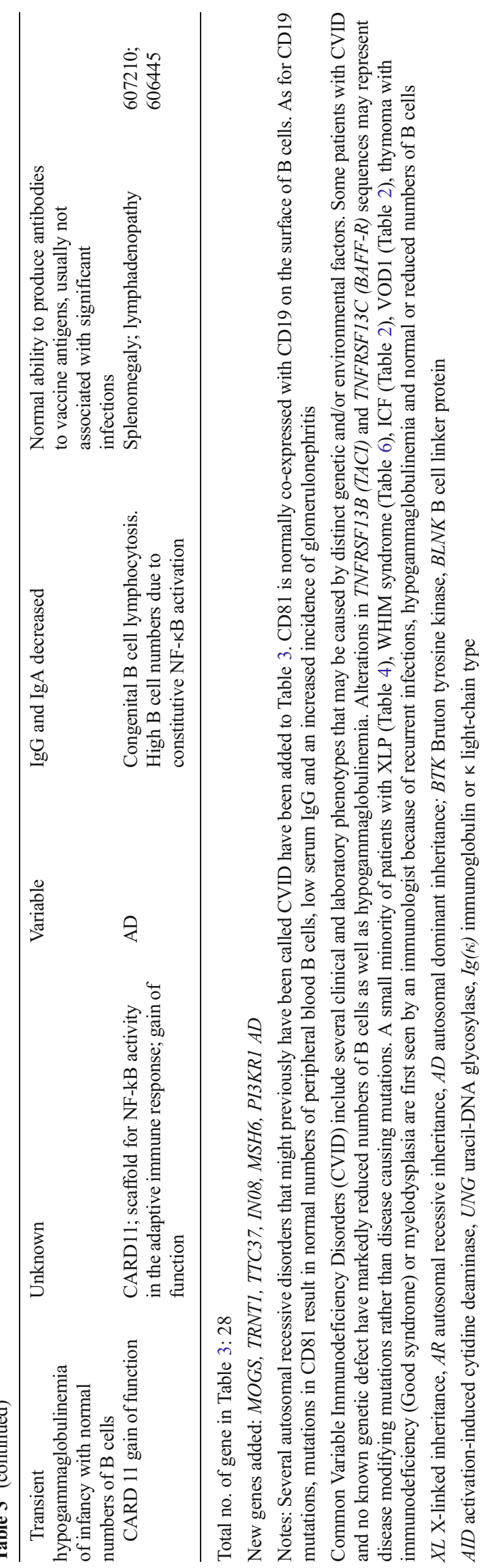




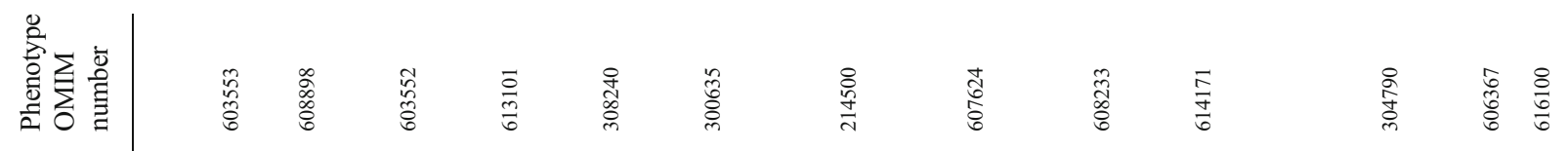

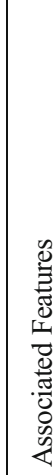
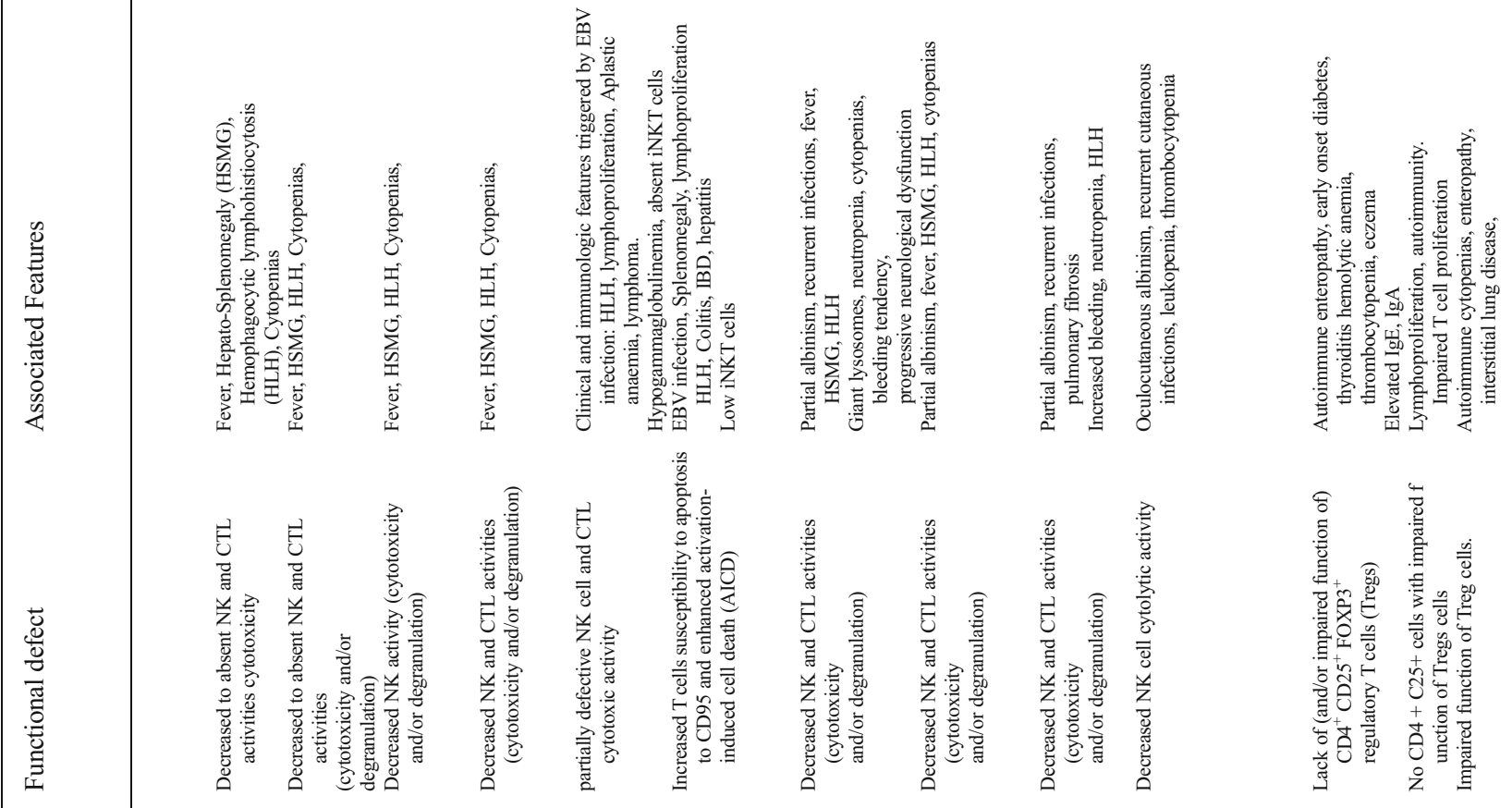

总

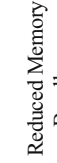

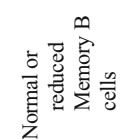

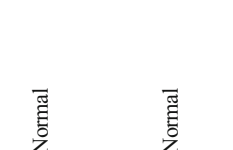

$1 \sqrt{1}$

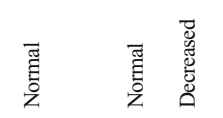

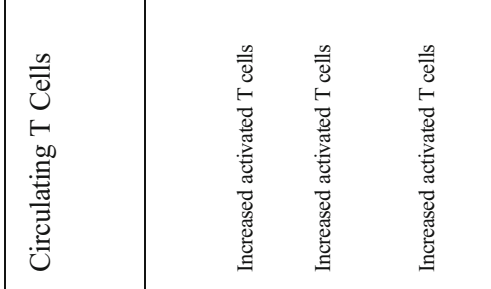
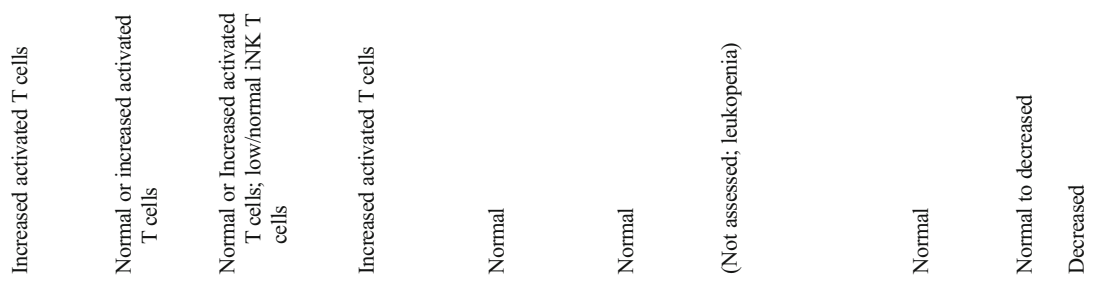

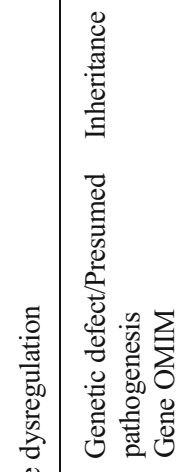

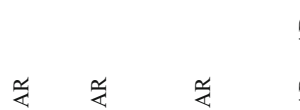

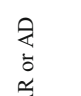

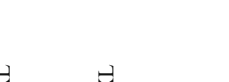

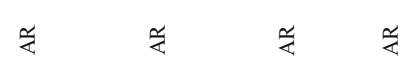

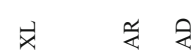

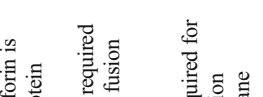

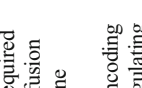

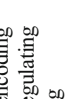

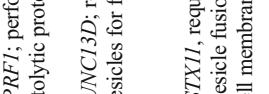

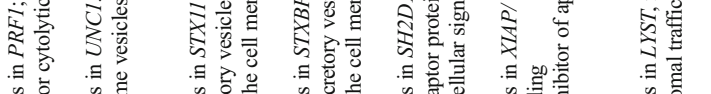

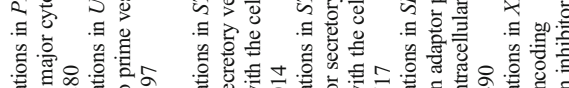

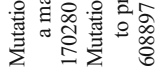

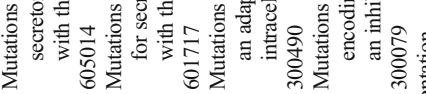

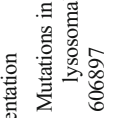

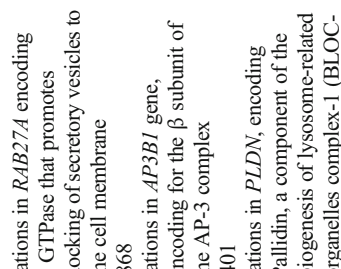

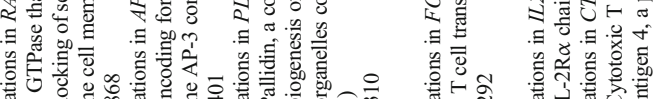

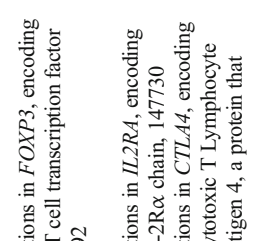

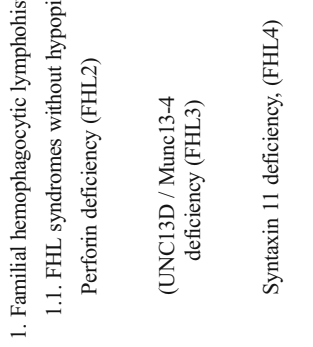

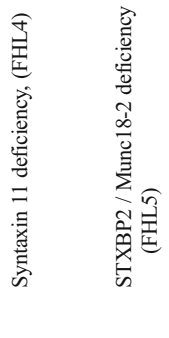

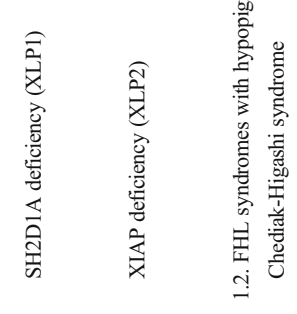

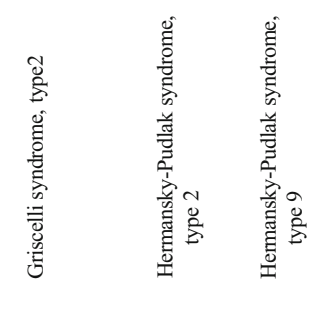

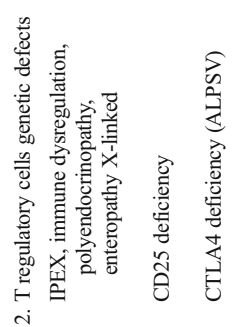




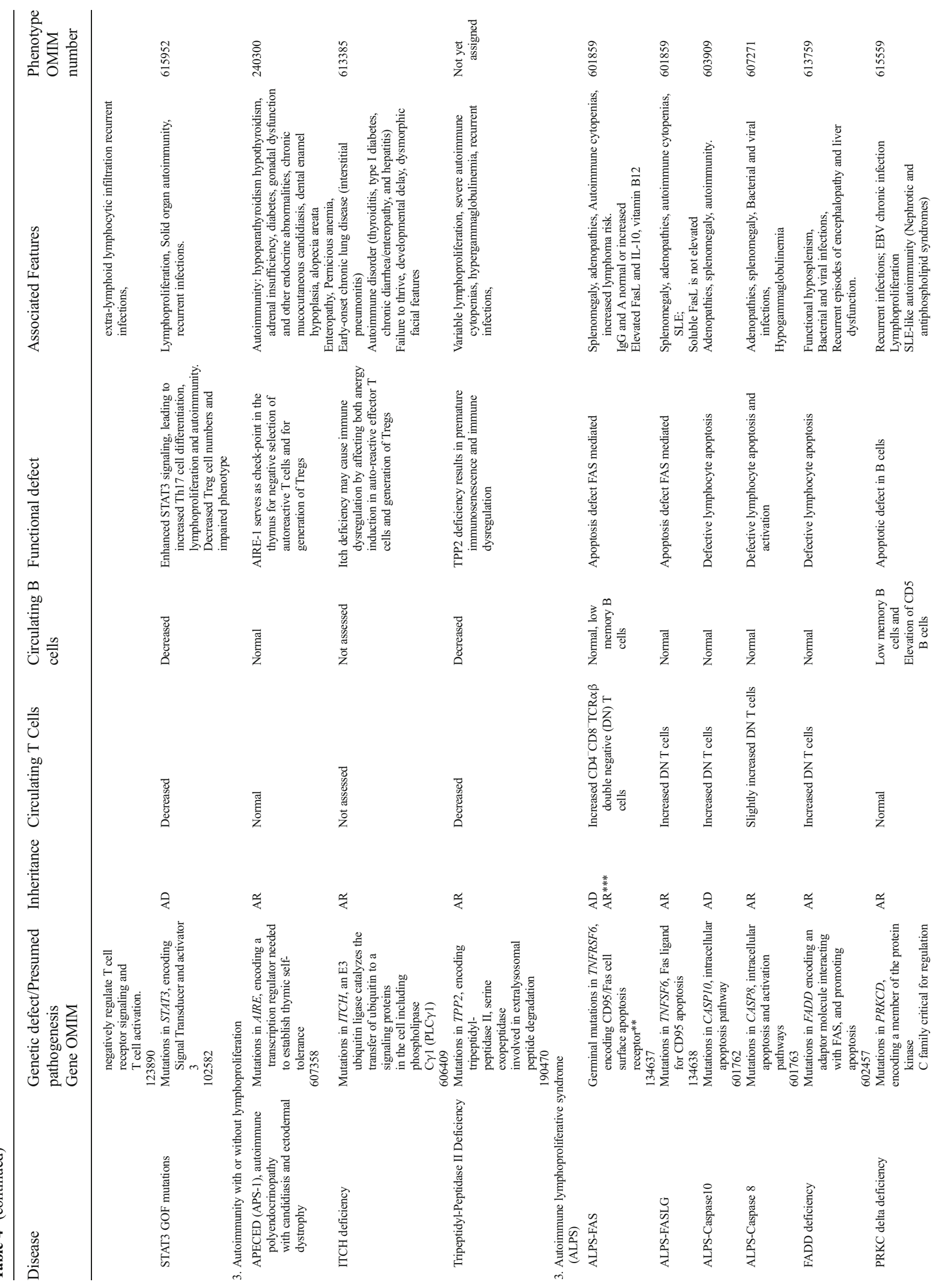




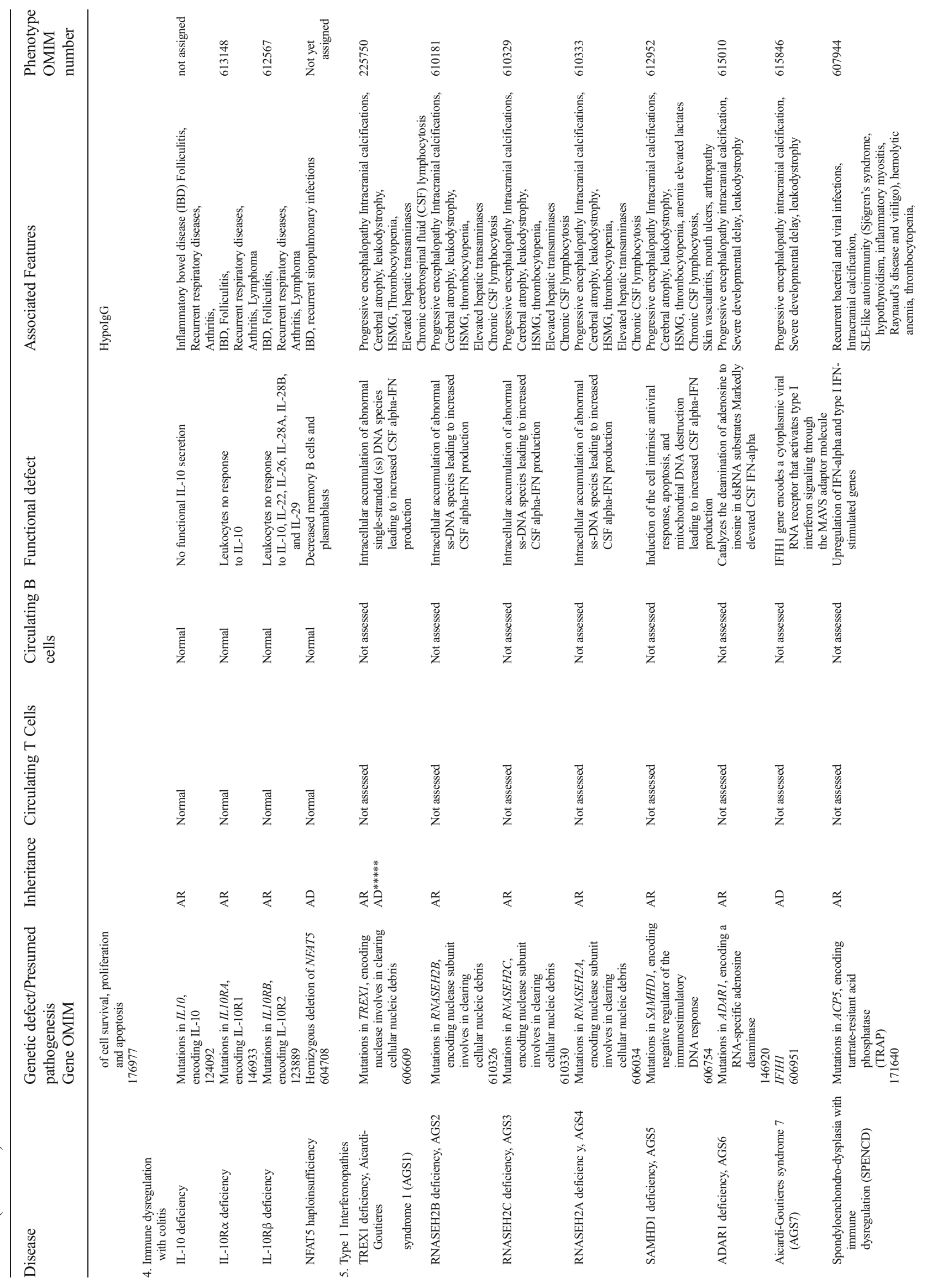




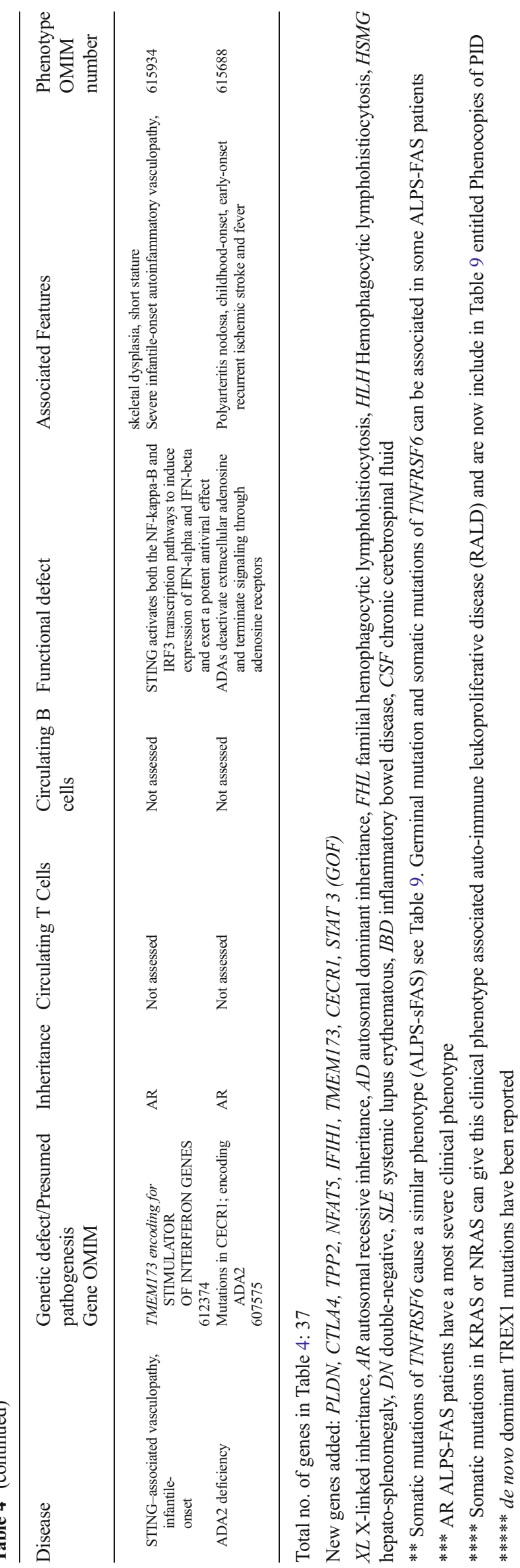




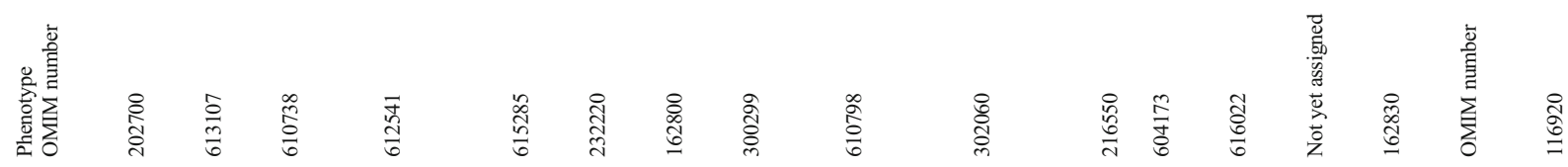

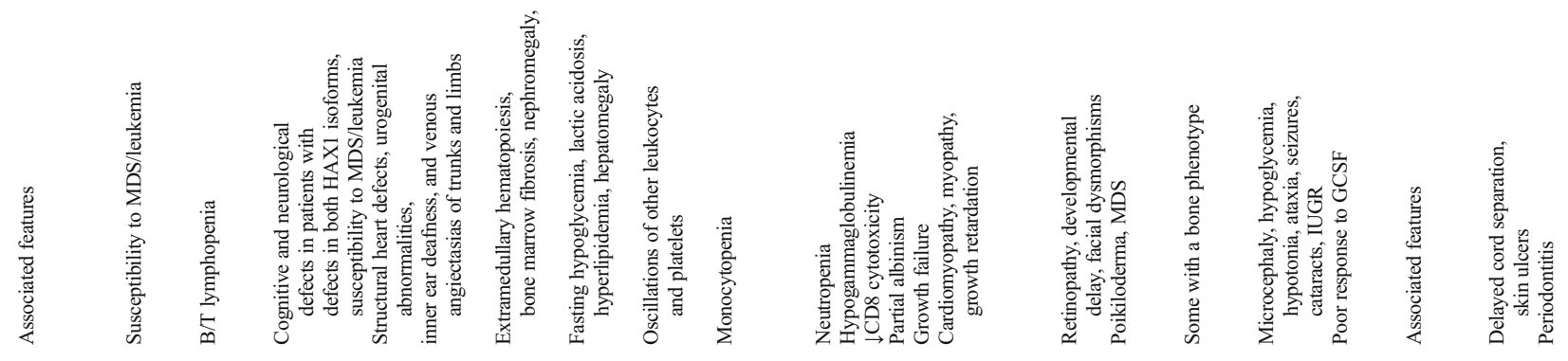

\|\|\|\|\|\|\|\|\|\|$\|$

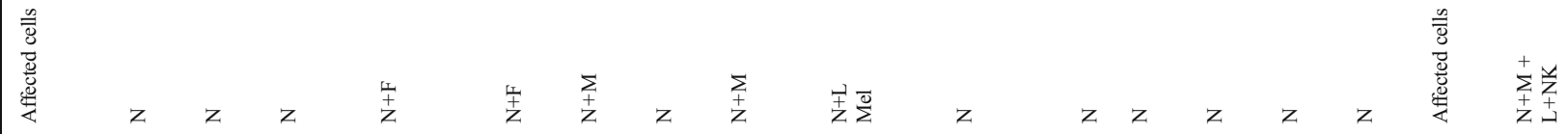




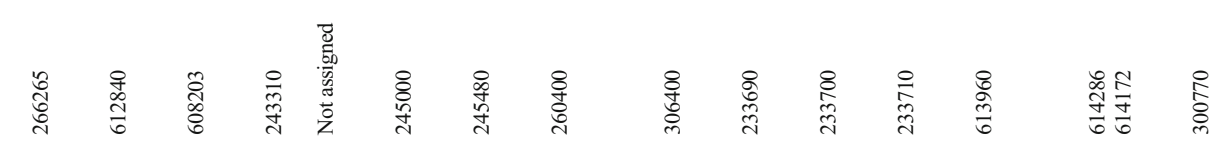

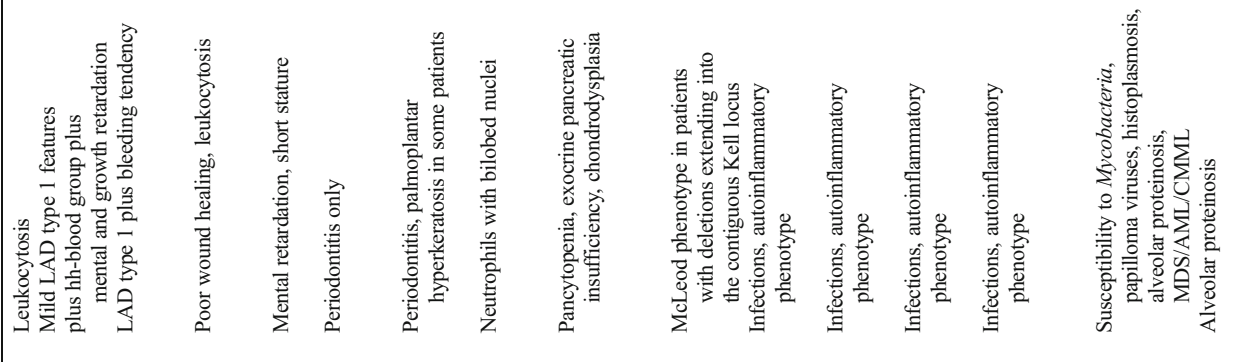

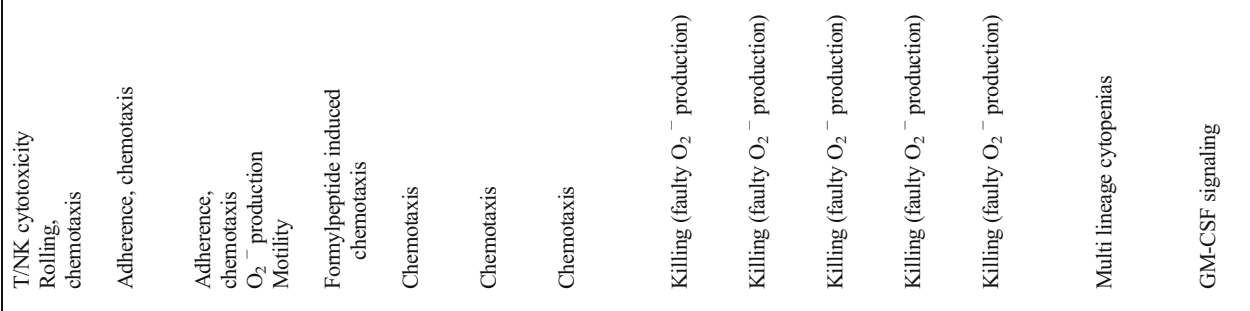

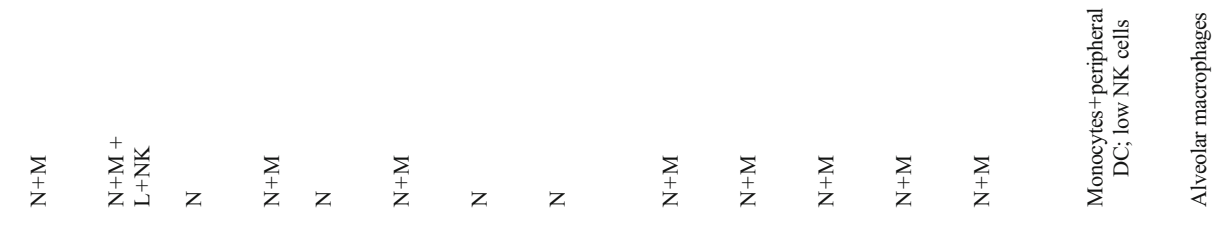

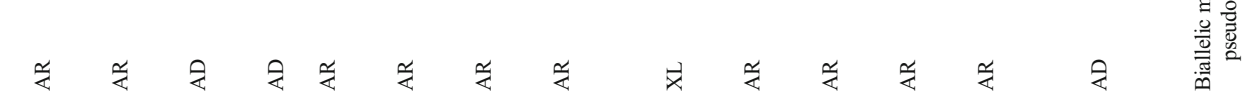

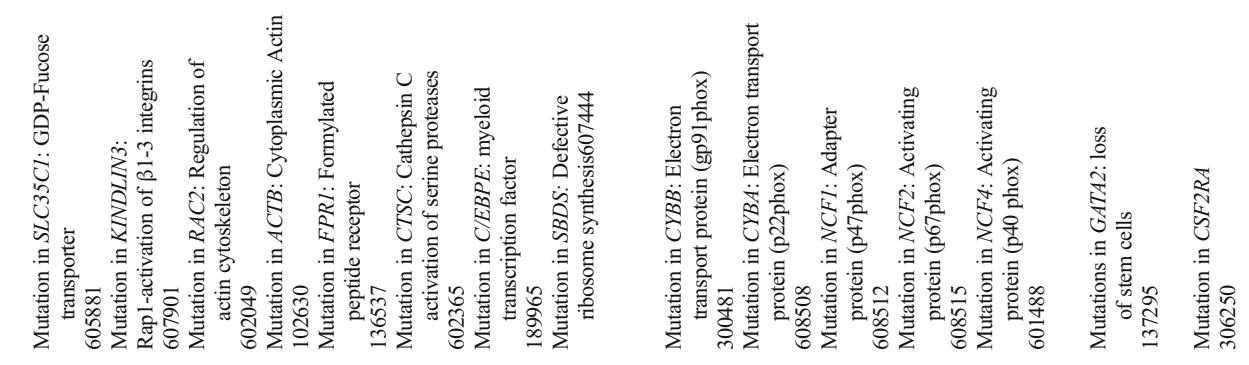

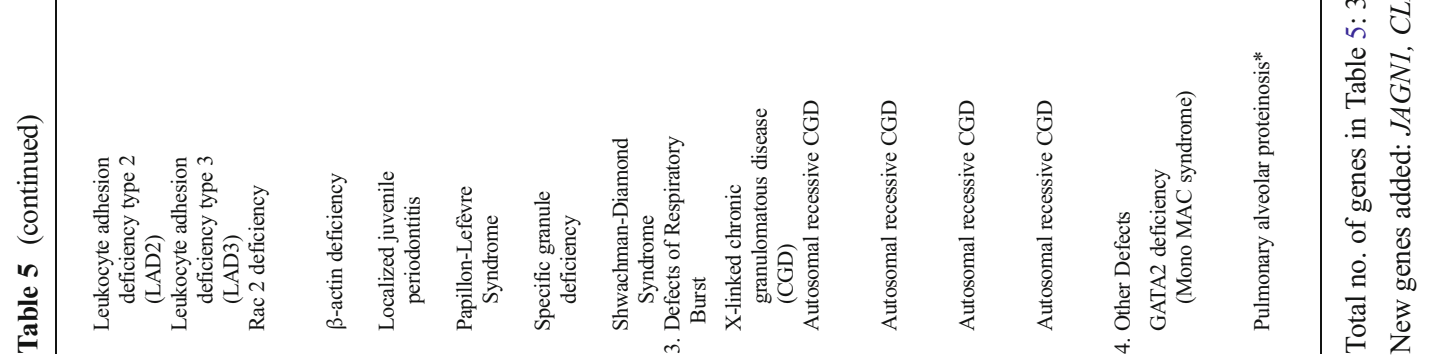




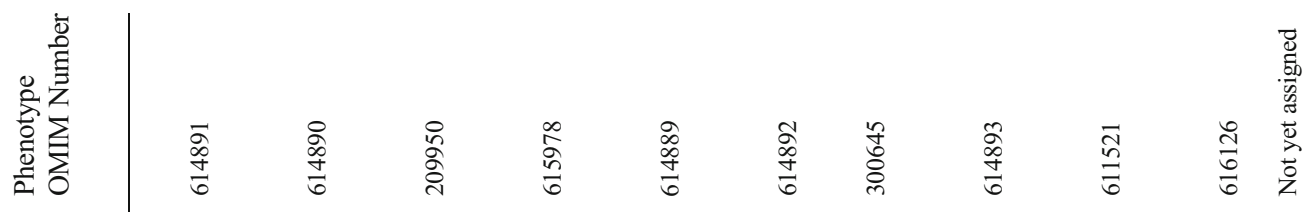

đัd

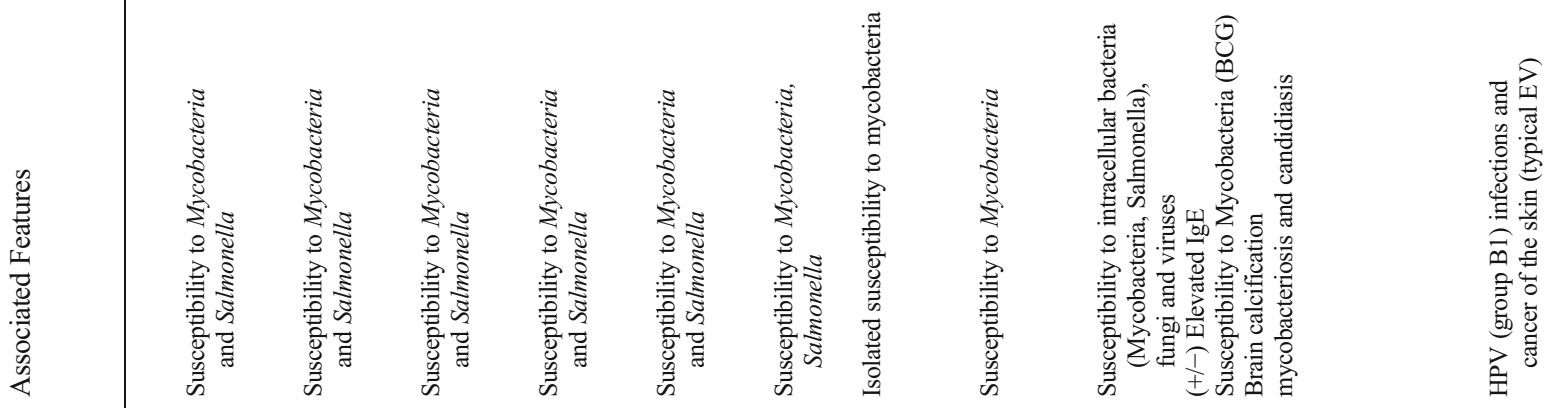

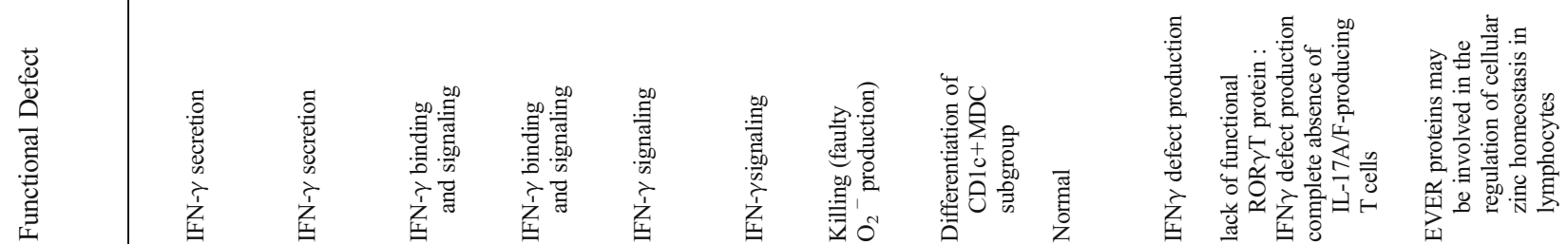

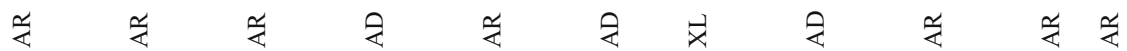

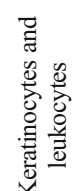

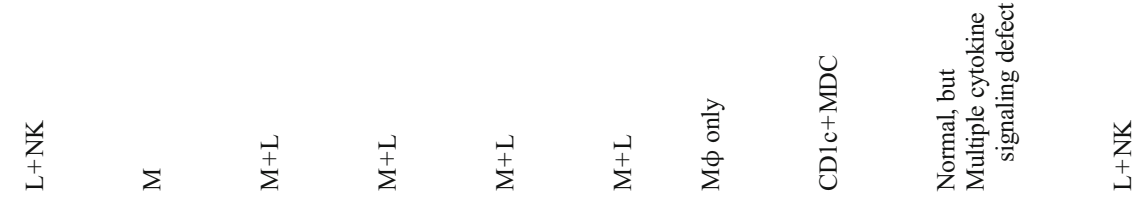

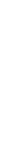

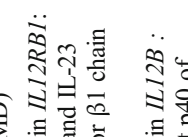

年我

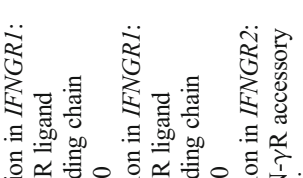

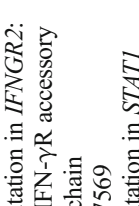

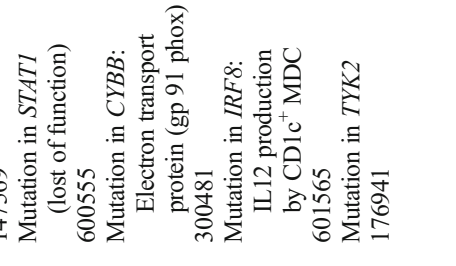

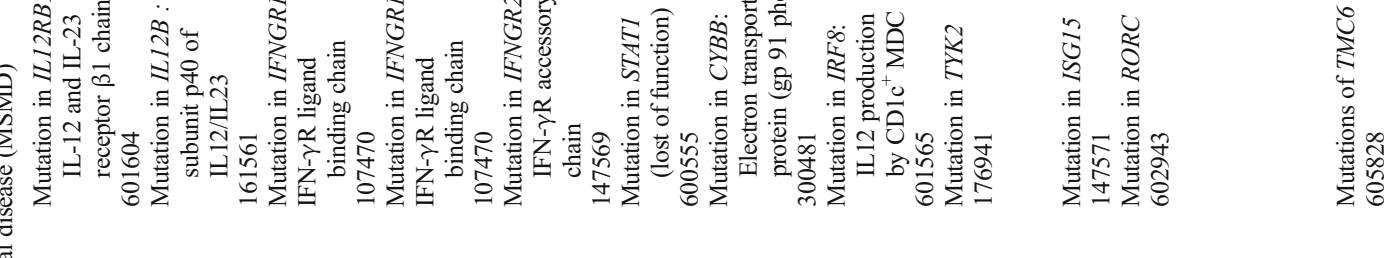

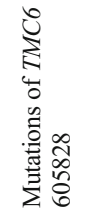

$\stackrel{2}{4}$ 


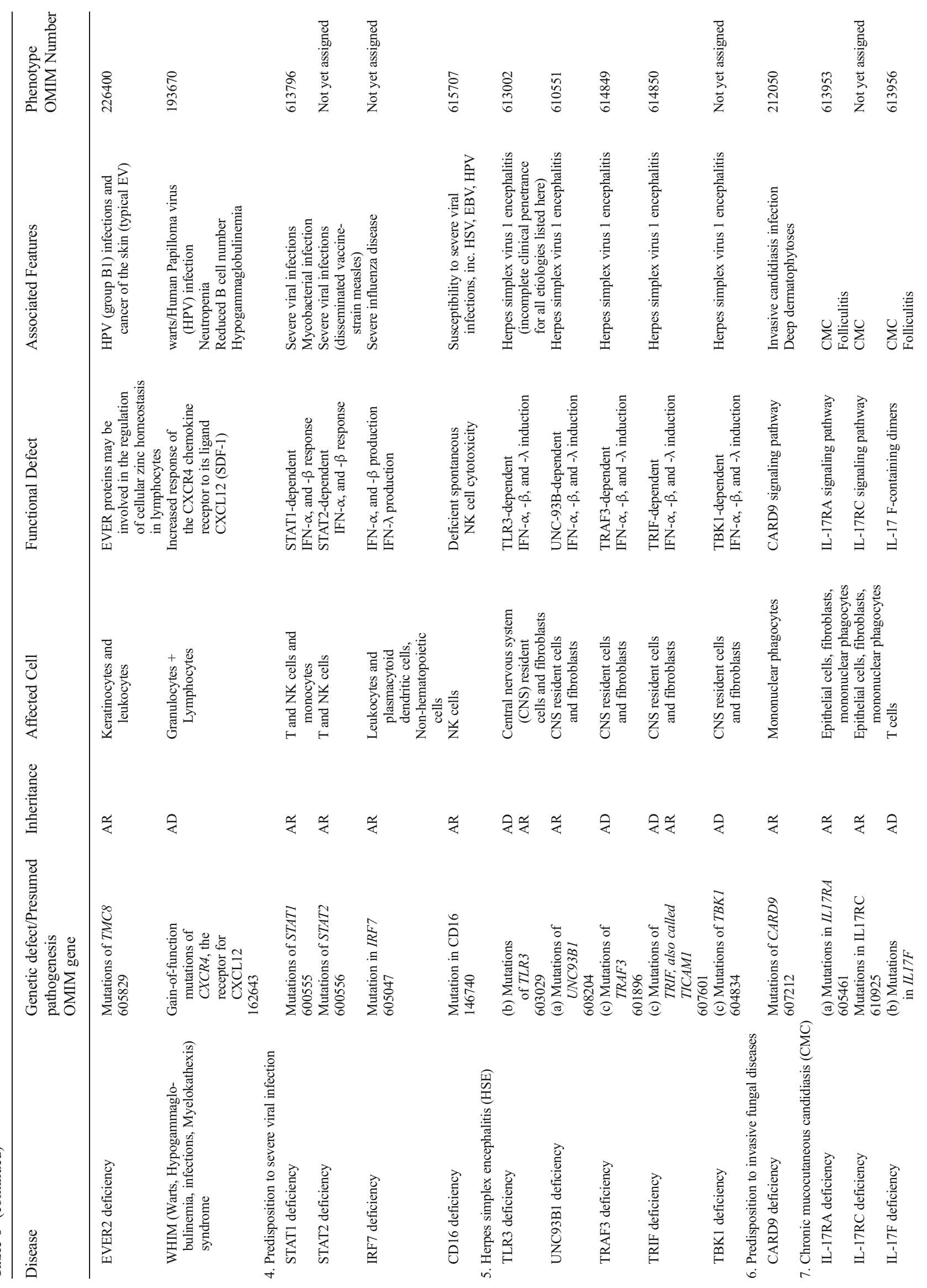




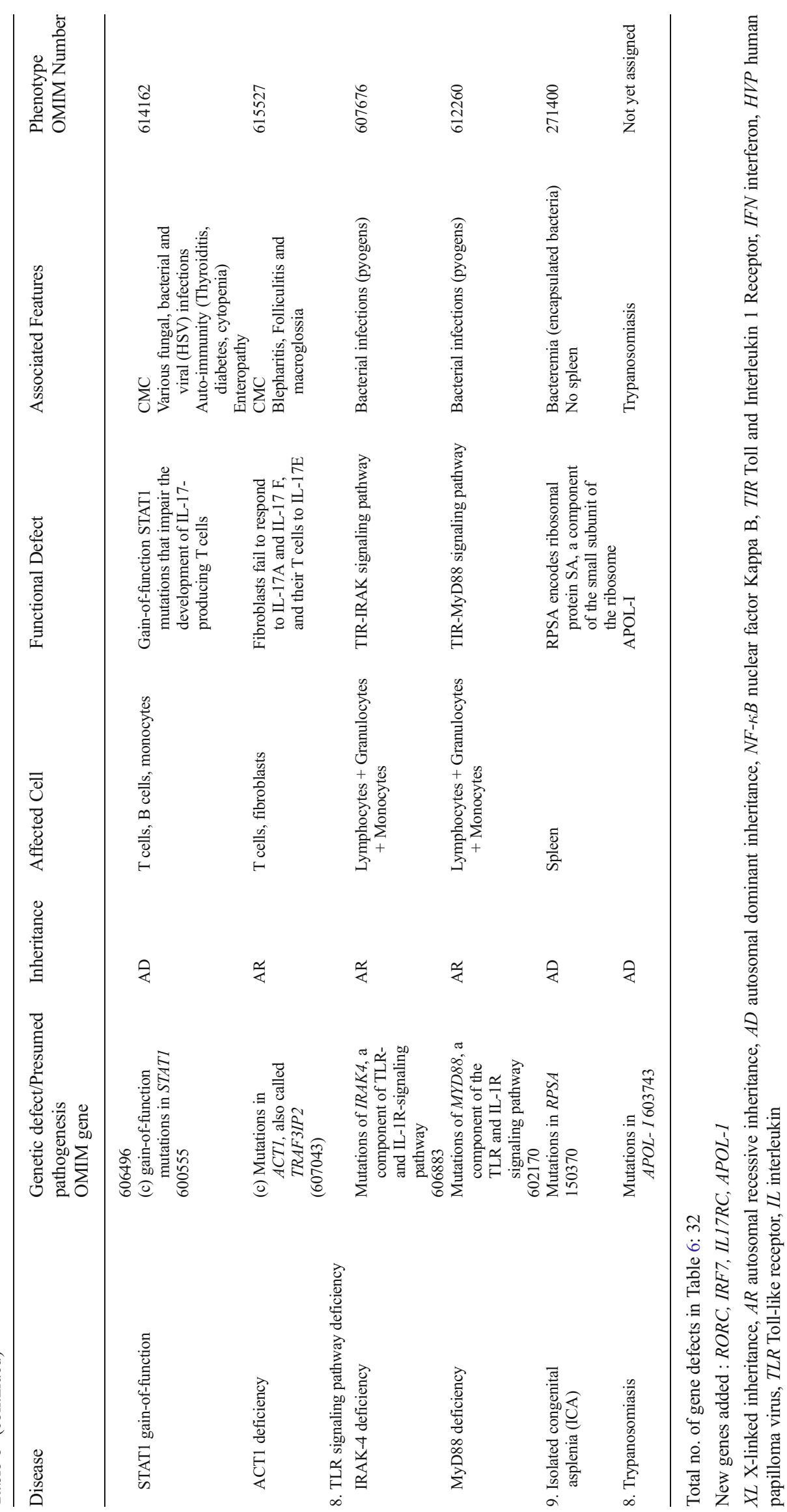



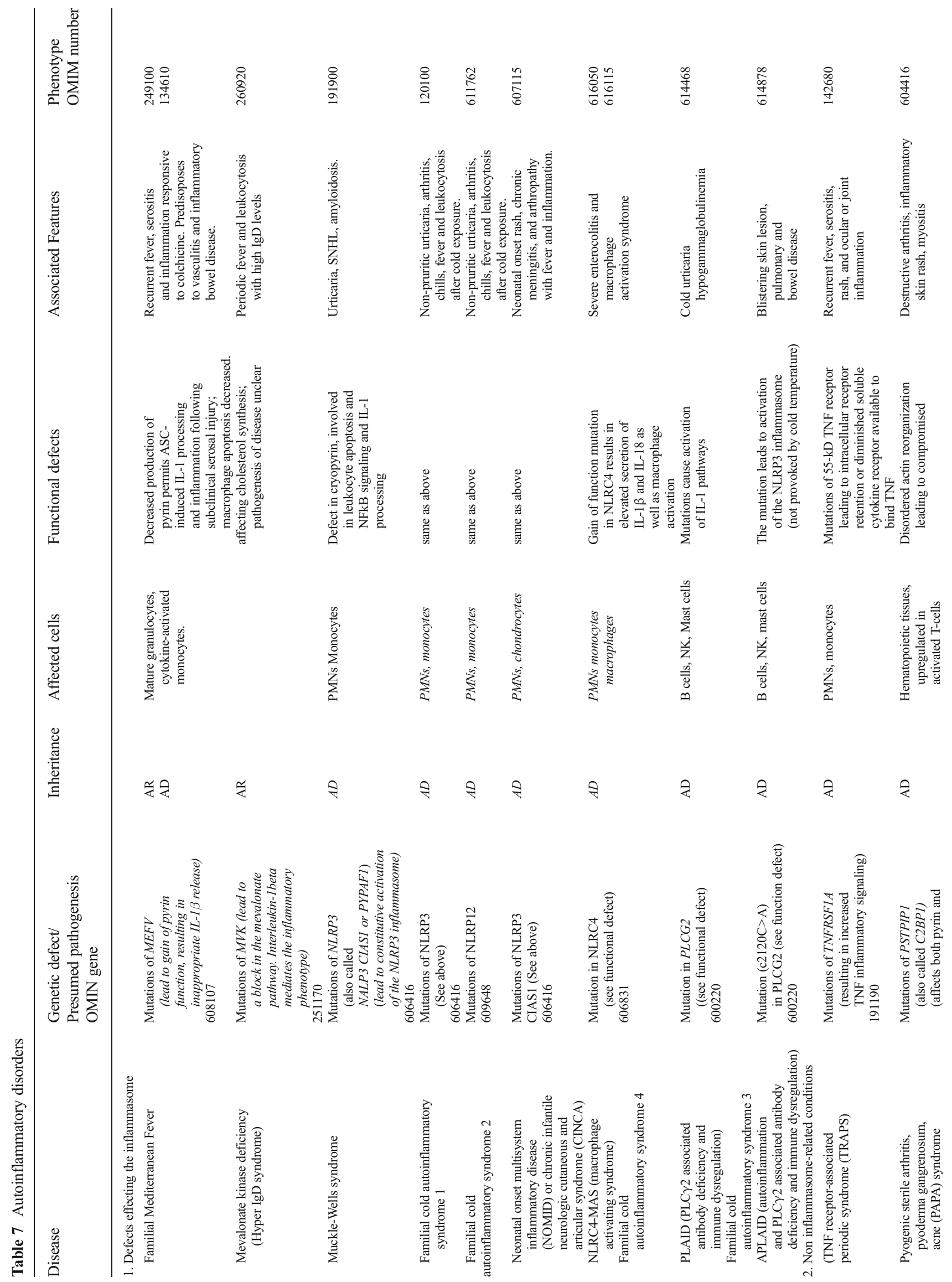


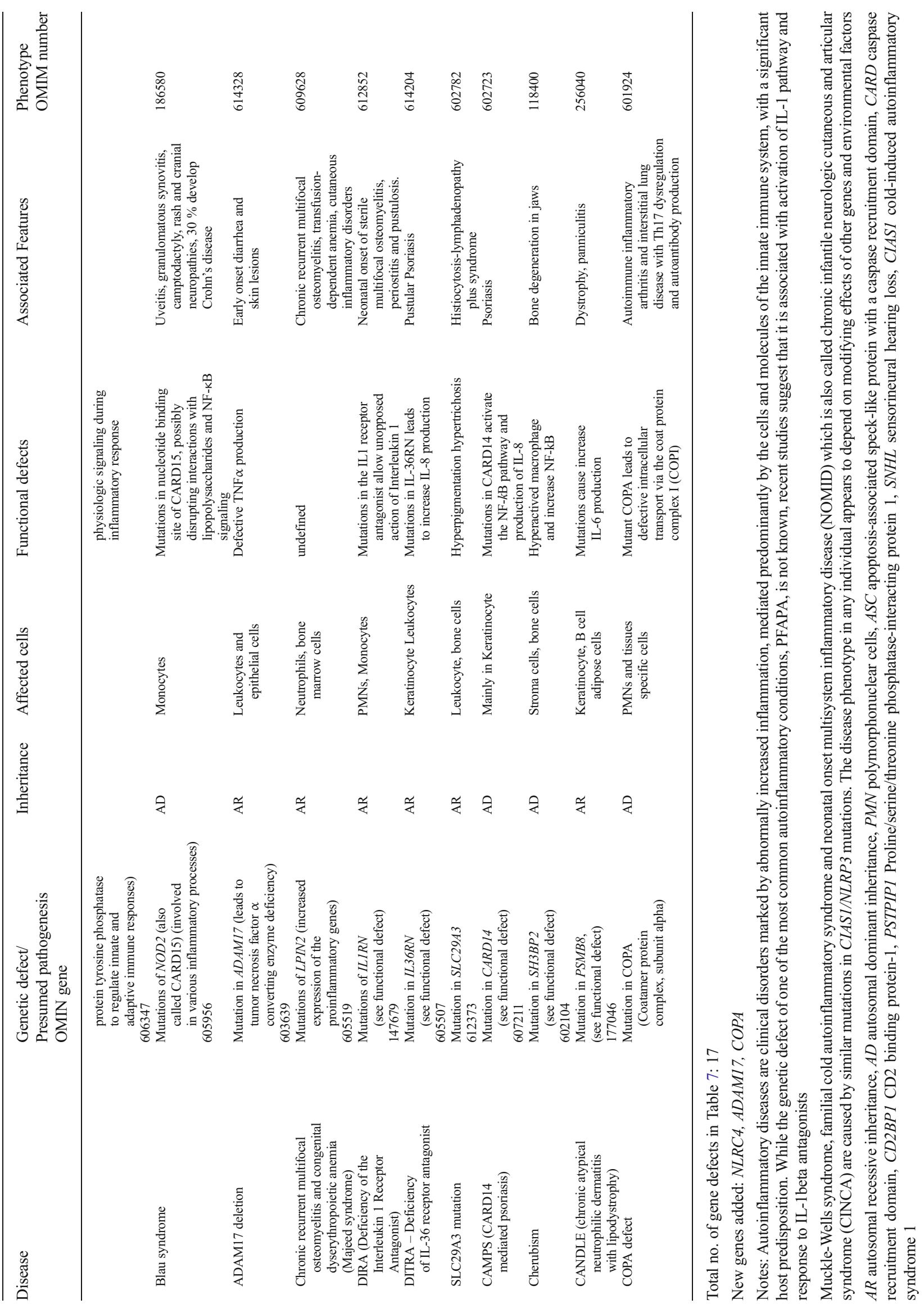




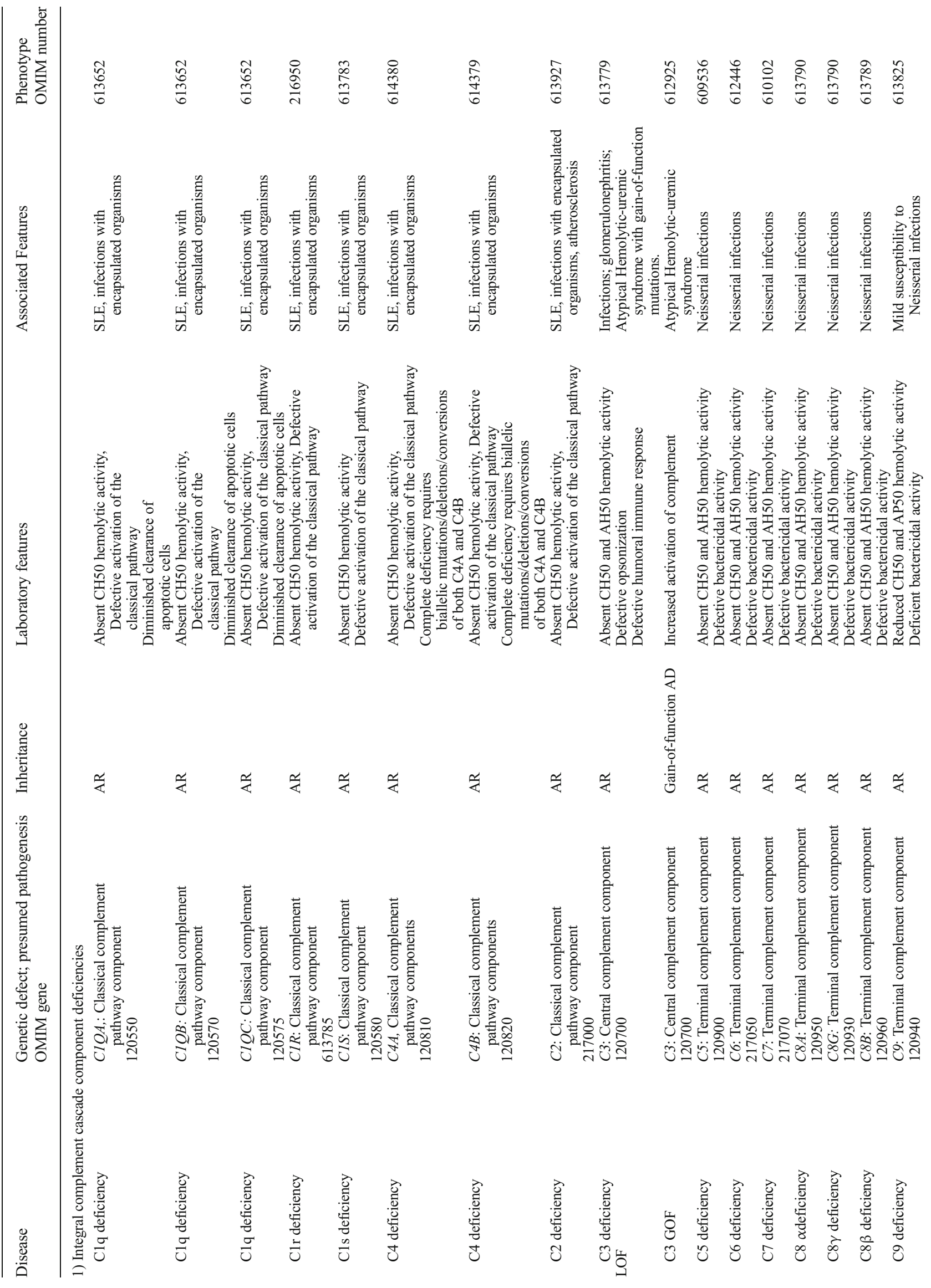



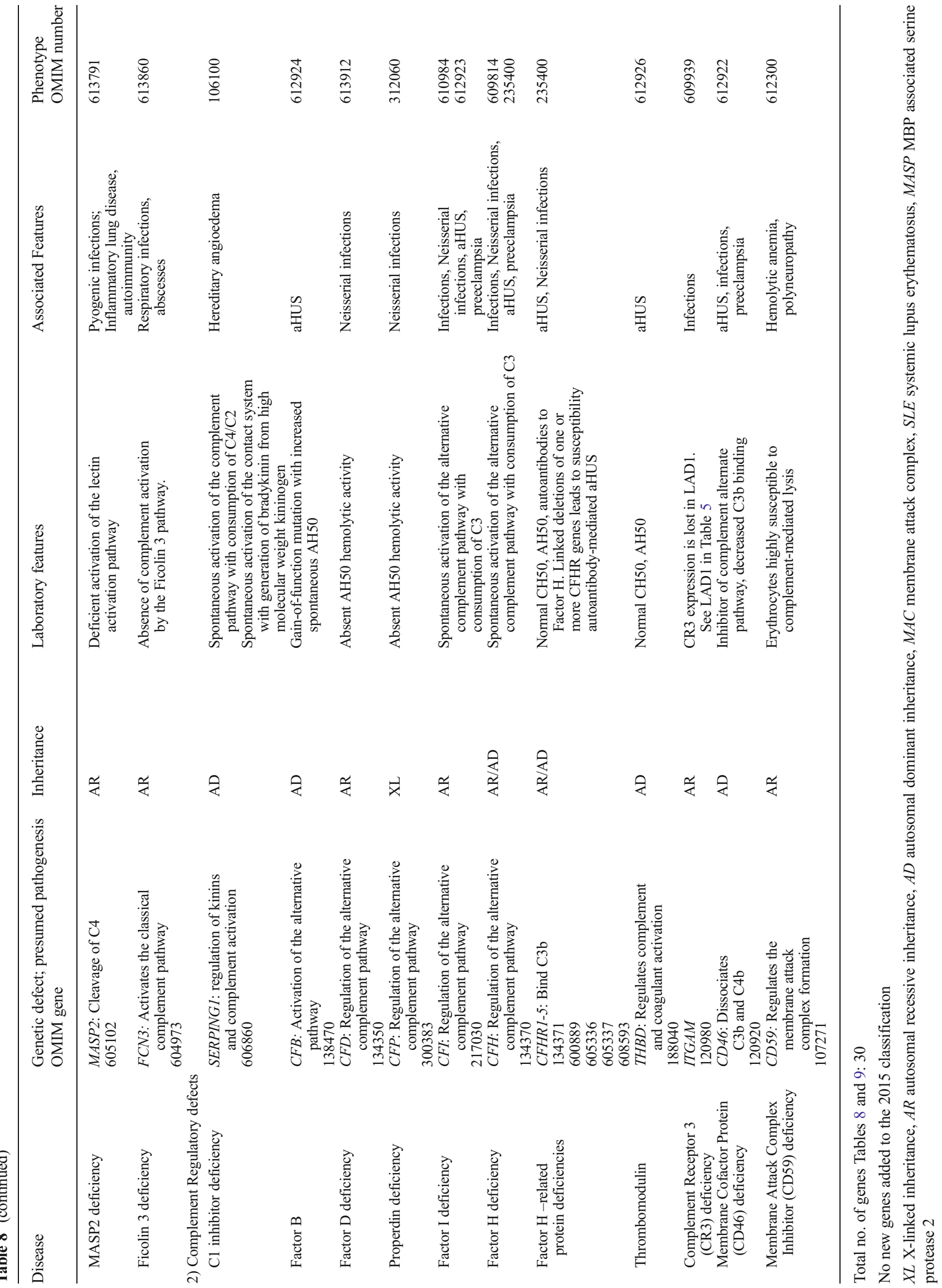


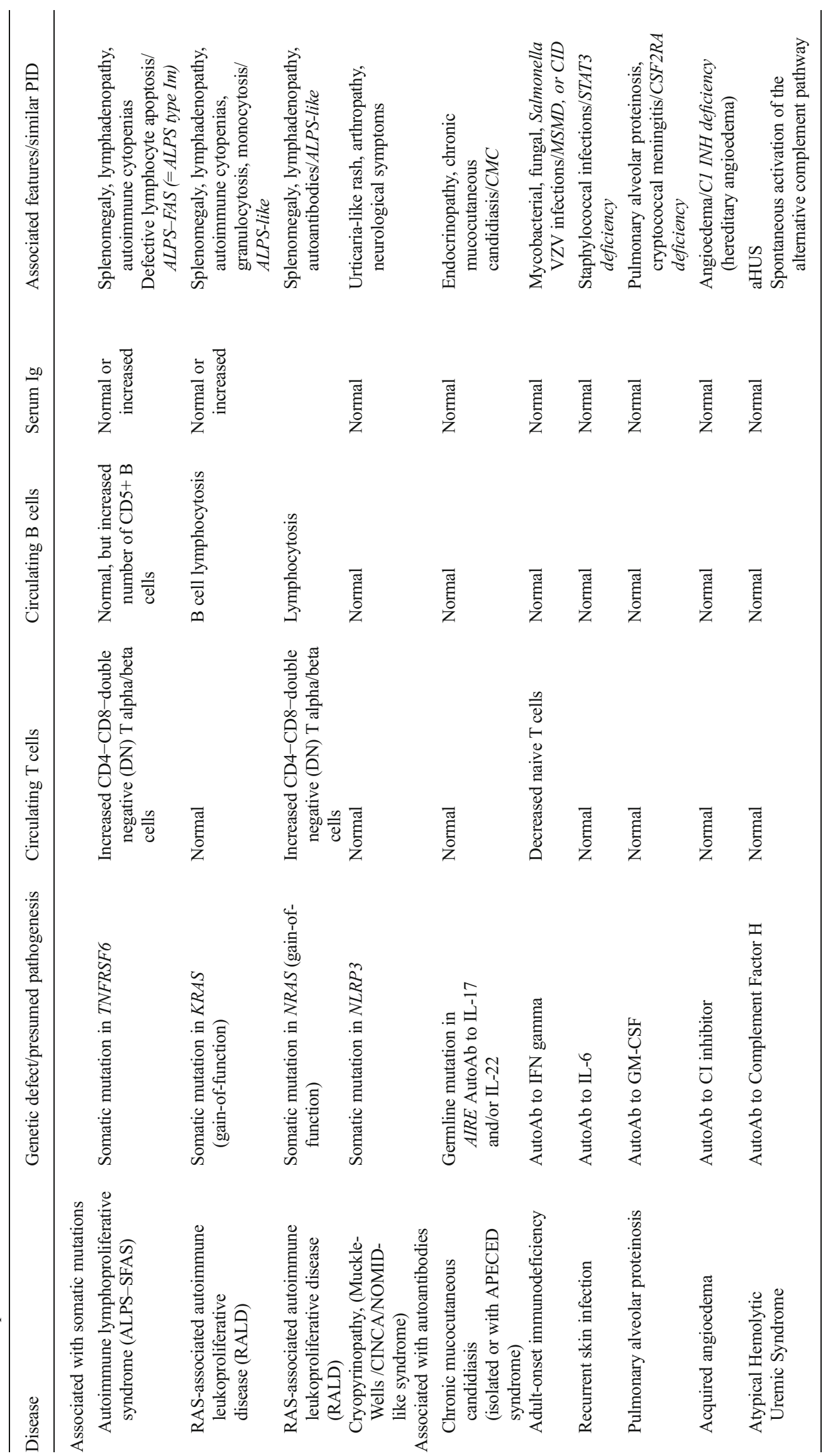


Open Access This article is distributed under the terms of the Creative Commons Attribution 4.0 International License (http:// creativecommons.org/licenses/by/4.0/), which permits unrestricted use, distribution, and reproduction in any medium, provided you give appropriate credit to the original author(s) and the source, provide a link to the Creative Commons license, and indicate if changes were made. 